\title{
Atmospheric pressure gas chromatography-time-of-flight-mass spectrometry (APGC-ToF-MS) for the determination of regulated and emerging contaminants in aqueous samples after stir bar sorptive extraction (SBSE)
}

\author{
Marina G. Pintado-Herrera, Eduardo González-Mazo, Pablo A. Lara-Martín* \\ Physical Chemistry Department, Faculty of Marine and Environmental Sciences, University of Cadiz, Campus de Excelencia Internacional del Mar (CEIMAR), \\ Cadiz 11510, Spain
}

\section{H I G H L I G H T S}

- 102 regulated and emerging contaminants are simultaneously extracted by SBSE.

- APGC-ToF-MS is presented as an alternative to EI for the analysis of microcontaminants.

- Identification of non-target compounds in environmental matrices is also explored.

\section{GRA P H I C A L A B S T R AC T}

Examples of identification of target (triclosan, TCS) and non-target (tributhyl phosphate, TBP) compounds in environmental water samples by APGC-ToF-MS. Some confirmation criteria are shown (i.e. mass spectra, isotopic distribution, and accurate mass).

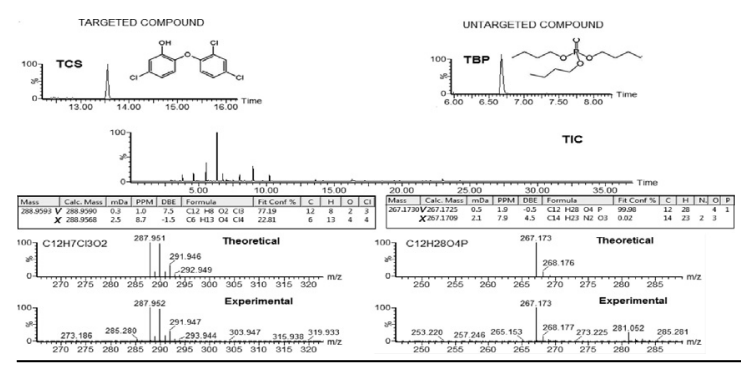

\begin{abstract}
A B S T R A C T
This work presents the development, optimization and validation of a multi-residue method for the simultaneous determination of 102 contaminants, including fragrances, UV filters, repellents, endocrine disruptors, biocides, polycyclic aromatic hydrocarbons (PAHs), polychlorinated biphenyls (PCBs), and several types of pesticides in aqueous matrices. Water samples were processed using stir bar sorptive extraction (SBSE) after the optimization of several parameters: agitation time, ionic strength, presence of organic modifiers, $\mathrm{pH}$, and volume of the derivatizing agent. Target compounds were extracted from the bars by liquid desorption (LD). Separation, identification and quantification of analytes were carried out by gas chromatography (GC) coupled to time-of-flight (ToF-MS) mass spectrometry. A new ionization source, atmospheric pressure gas chromatography (APGC), was tested. The optimized protocol showed acceptable recovery percentages (50-100\%) and limits of detection below $1 \mathrm{ng} \mathrm{L}{ }^{-1}$ for most of the compounds. Occurrence of 21 out of 102 analytes was confirmed in several environmental aquatic matrices, including seawater, sewage effluent, river water and groundwater. Non-target compounds such as organophosphorus flame retardants were also identified in real samples by accurate mass measurement of their molecular ions using GC-APGC-ToF-MS. To the best of our knowledge, this is the first time that this technique has been applied for the analysis of contaminants in aquatic systems. By
\end{abstract}

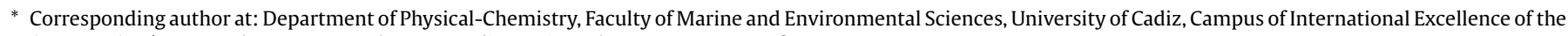
Sea (CEI.MAR), Río San Pedro, Puerto Real, 11510 Cadiz, Spain. Tel.: +34 956016159; fax: +34 95601040

E-mail address: pablo.lara@uca.es (P.A. Lara-Martín).
} 
employing lower energy than the more widely used electron impact ionization (EI), AGPC provides significant advantages over EI for those substances very susceptible to high fragmentation (e.g., fragrances, pyrethroids).

(c) 2014 Elsevier B.V. All rights reserved.

\section{Introduction}

The production and release of synthetic chemicals into the environment have been constantly increasing over the last 50 years due to the exponential growth of the world population and industrialization. Thousands of different organic compounds are discharged into aquatic systems every day from point (e.g., urban and industrial wastewater discharges) [1] and diffuse pollution sources (e.g., use of fertilizers in agriculture or atmospheric deposition of pollutants from vehicles and factories) [2]. So far, only a few of these contaminants have been studied in detail and regulated based on their well-known toxicity and/or other negative environmental impacts (Stockholm Convention on Persistent Organic Pollutants, 1991) [3]. Examples are polycyclic aromatic hydrocarbons (PAHs), pesticides, and other priority pollutants such as polybrominated diphenyl ethers. Most recently, the scientific community has been targeting new compounds [4] - so called emerging contaminants - that are less persistent but, due to their chemical structure and continuous input into the environment, may disrupt endocrine and reproductive systems, alter animal behavior and damage tissues [5-7]. One of the most important groups in terms of production volume are personal care products (PCPs), which include fragrances, sun-screen agents and antiseptics, surfactants and some new flame retardants, among others chemicals. Risk assessments and/or ecotoxicity data do not exist for most of these contaminants.

Recent attempts to control and prevent contamination of aquatic ecosystems, such as the European Water Policy Directive 2008/105/EC [8], have established very restrictive Environmental Quality Standards (EQS) for many of the compounds mentioned above, restricting their presence in water bodies to concentrations of less than a few ppb or ppt. The demand for new, sensitive, reliable and cost-effective multiresidue analytical methods for a large number of compounds is therefore growing. Due to the low levels that must be measured, most methods rely on preconcentration techniques before analysis. Solid phase extraction (SPE) or liquid-liquid extraction (LLE) are often employed [9,10], although, over the last decade, the use of sorptive techniques such as stir bar sorptive extraction (SBSE) or solid-phase microextraction (SPME) has increased. Using polymers such as polydimethylsiloxane (PDMS) that can adsorb moderately to highly hydrophobic contaminants, sorptive techniques minimize sample volume and manipulation, as well as the use of organic solvents, thus achieving lower detection limits than more conventional approaches [11,12]. After extraction, target compounds are often released from the polymer using thermodesorption (TD) units coupled to gas chromatography [13-15]. If TD is not feasible and/or better results are achieved by liquid chromatography, a minimal amount of solvent can be used instead to desorb the analytes (liquid desorption, or LD) $[14,16,17]$.

After preconcentration, analysis of most volatile nonpolar or semi-polar contaminants in water samples is usually performed by gas chromatography-quadrupole-mass spectrometry (GC-Q-MS) $[9,16]$ or by tandem mass spectrometry (GC-MS/MS) $[12,18]$. Most analytes are ionized and fragmented by electron impact (EI) ionization. Due to the complex nature of many environmental matrices and low concentrations of target compounds, higher selectivity and sensitivity are often required, so scanning for MS/MS transitions using triple quadrupole mass analyzers (TQ) is preferred. As a drawback, the number of compounds that can be detected in this mode is limited. More recently, a new ionization source named atmospheric pressure gas chromatography (APGC) has been developed. The ionization process takes place by means of charge transfer $\left(\mathrm{M}^{+}\right)$or protonation $\left(\mathrm{M}+\mathrm{H}^{+}\right)$, using a reagent gas (nitrogen) and lower energy than EI so that fragmentation of molecular ions is lower. So far, APGC has only been coupled to time-of-flight (ToF) analyzers, improving not only the sensitivity but also the mass resolution achieved in full scan mode by more conventional GC-Q-MS or GC-TQ-MS. Applications using APGC are still scarce. Some examples are determination of PAHs and nitropolycyclic aromatic hydrocarbons (NPAH) in biological samples (mosses), as well as the analysis of acrylic adhesives [19], fruits [20], and petroleum [21]. To the best of our knowledge, there are no published studies on determination of organic contaminants in the environment using GC-APGC-ToF-MS, which could be used as an alternative to GC-MS/MS, especially for the screening of unknowns and to avoid false positives.

The aim of this study is to develop a new method for the extraction and determination of a wide range of regulated and emerging organic contaminants (102 compounds in total, including several types of pesticides, PCBs, PAHs, endocrine disruptor compounds, fragrances and UV filters) in water bodies. We have focused our attention primarily on: (a) optimizing key parameters (e.g., $\mathrm{pH}$, ionic strength, derivatization agents.) for the extraction of target compounds using SBSE; (b) exploring the possibilities of the analysis of organic contaminants by GC-APGC-ToF-MS; and (c) applying the developed methodology to the analysis of target (and also non-targeted) compounds in different environmental aqueous matrices.

\section{Experimental}

\subsection{Materials and reagents}

Methanol, acetonitrile, dichloromethane and ethyl acetate were of chromatography quality, purchased from Sigma-Aldrich (Madrid, Spain). Sodium chloride was purchased from Merck and water was HPLC gradient grade, from J.T. Baker (The Netherlands). Acetic anhydride, used as derivatizing agent and sodium carbonate were purchased from Sigma-Aldrich (Madrid, Spain). Commercial PDMS stir bars (size $10 \mathrm{~mm} \times 0.5 \mathrm{~mm}$ and $20 \mathrm{~mm} \times 0.5 \mathrm{~mm}$, length $\times$ film thickness) and a 15-position magnetic stirrer was purchased from Gerstel (Mulheim a/d Ruhr, Germany). Glass microfiber filters $(0.45 \mu \mathrm{m}$ pore size) were purchased from Pall Corporation (Michigan, United States). Data were processed using MassLynx 4.1 software, and Mass Fragment was used occasionally to determine fragmentation patterns for selected compounds.

\subsection{Chemicals}

$N, N$-diethyl-meta-toluamide (DEET), galaxolide, $17-\beta$ estradiol (E2), mixtures of polycyclic aromatic hydrocarbons (PAHs) (acenaphthylene, acenaphthene, fluorene, anthracene, phenanthrene, pyrene, fluoranthene, benzo[a]anthracene, chrysene, benzo[ $b]$ fluoranthene, benzo[ $k]$ fluoranthene, benzo[a]pyrene, dibenzo[ $a, h]$ anthracene, indeno[1,2,3-cd]pyrene, benzo[g,h,i]perylene), triazines (atraton, ametryn, atrazine, prometon, 
secbumeton, prometryn, terbutryn, propazine, terbuthylazine, simazine and simetryn), organochlorine pesticides (lindane, heptachlor, heptachlor epoxide isomer $B, \alpha$-endosulfan, $\beta$-endosulfan, $\alpha$-chlordane, $\gamma$-chlordane, $p, p^{\prime}$-DDT, $o, p^{\prime}$-DDT, $p, p^{\prime}$-DDE, endosulfan sulfate, dieldrin, endrin, methoxychlor, endrin ketone and aldrin), organophosphorus pesticides (parathion, ethion and carbophenothion and chlorpyrifos), polychlorinated biphenyls (PCB28, PCB52, PCB138, PCB153, PCB180 and PCB101), and pyrethroids (bifenthrin, phenothrin, permethrin, deltamethrin, fenvalerate, cypermethrin and cyfluthrin) were purchased from $\mathrm{Dr}$ Ehrenstorfer GmbH (Augsburg, Germany), as well as triclosan d3 (TCS-d3), terbuthylazine $\mathrm{d} 5$, musk xylene $\mathrm{d} 15$, methyl-triclosan ${ }^{13} \mathrm{C}_{12}$ (MTCS- ${ }^{13} \mathrm{C}_{12}$ ), and a deuterated PAH mixture (chrysene $\mathrm{d} 12$, phenanthrene $\mathrm{d} 10$ and perylene d12). Oxybenzone (BP-3), octocrylene $(\mathrm{OC})$, nonylphenol technical mixture (NP), bisphenol A (BpA), estrone (E1), 17 $\alpha$-ethynylestradiol (EE2), musk xylene (MX), musk ketone (MK), musk moskene (MM), triclosan (TCS), methyl-triclosan (MTCS), octylphenol (OP), mexenone (BP-10), avobenzone, 2-hydroxybenzophenone (2-OHBP), 3-hydroxybenzophenone (3-OHBP), 4-hydroxybenzophenone (4-OHBP), 2-ethylhexyl 4-dimethylaminobenzoate (OD-PABA), homosalate (HMS), benzyl salicylate (BS), 2-ethylhexyl salicylate (EHS), 2-ethylhexyl-4-methoxycinnamate (EHMC), 4-methylbenzylidene camphor (4-MBC), and benzophenone d10 were purchased from Sigma-Aldrich (Madrid, Spain). Celestolide, tonalide, traseolide, phantolide, musk tibetene (MT), musk ambrette (MA), cashmeran, and irgarol were purchased from LGC Standards (Barcelona, Spain). Helvetolide, habanolide, exaltolide, muscone, exaltenone and muscenone delta were purchased from Firmenich (Barcelona, Spain). OTNE fragrance was purchased from Bordas Chinchurreta Destilaciones (Seville, Spain) and musk R1 from Yingkou Tanyun Chemical Research Institute (Yingkou, China). Stock solutions of these analytes were prepared in methanol and stored at $-20^{\circ} \mathrm{C}$ in tightly closed amber vials.

\subsection{Sample collection}

Several aqueous samples were collected in autumn 2012 from different areas in the province of Cadiz (SW Spain). Sewage samples were collected from the effluent of a wastewater treatment plant (WWTP) in Puerto Real (40,000 inhabitants). The facility, equipped with biologic treatment, treats the sewage coming from this town, receiving an average flow of $8000 \mathrm{~m}^{3} \mathrm{~d}^{-1}$ and discharging into the Bay of Cadiz. Surface seawater samples were taken from the Rio San Pedro tidal channel, located within the natural park of Cadiz Bay (36 $\left.34^{\prime} 31.05^{\prime \prime} \mathrm{N} ; 6^{\circ} 12^{\prime} 37.80^{\prime \prime} \mathrm{W}\right)$. River water samples were collected from Guadalete river $\left(36^{\circ} 38^{\prime} 21.54^{\prime \prime}\right.$ $\mathrm{N} ; 6^{\circ} 7^{\prime} 46.75^{\prime \prime} \mathrm{W}$ ), downstream from the effluent of the WWTP in Jerez de la Frontera (200,000 inhabitants), which receives and treats wastewater not only from urban but also from agricultural and industrial sources. Groundwater samples from the Alluvial Guadalete aquifer (code 062.008) were obtained from a well. All water samples were collected in clean amber-glass bottles $(1000 \mathrm{~mL})$, filtered $(0.45 \mu \mathrm{m})$ and placed in a cooler at $4{ }^{\circ} \mathrm{C}$ prior to analysis, which was carried out within $24 \mathrm{~h}$.

\subsection{SBSE extraction and in situ derivatization (optimized procedure)}

Analytes were extracted from the water samples by SBSE. Optimization of the extraction was performed by spiking HPLC water aliquots $(10 \mathrm{~mL})$ with all target compounds at $1 \mathrm{ng} \mathrm{mL} \mathrm{m}^{-1}$. Surrogates (TCS d3, ${ }^{13} \mathrm{C}_{12}$-MTCS, chrysene $\mathrm{d} 12$, phenanthrene $\mathrm{d} 10$, perylene $\mathrm{d} 12$, bisphenol A d14, terbuthylazine $\mathrm{d} 5$ and benzophenone $\mathrm{d} 10)$ were also added to environmental samples $\left(0.1 \mathrm{ng} \mathrm{mL}^{-1}\right)$ to determine possible fluctuations during the extraction and analysis procedures. The influences of the agitation time, organic modifier, ionic strength, volume of derivatization agent and sample volume were evaluated by means of recovery experiments (percent of standard added to sample recovered during extraction). Recoveries were calculated in triplicate, and the background contamination was taken into account. Blanks consisted of nonspiked HPLC water that was extracted following the same procedure as that for samples. Prior to use, all PDMS stir bars were preconditioned by soaking them in a mixture of acetonitrile/ methanol $(80: 20, \mathrm{v} / \mathrm{v})$ overnight. Later, these bars were placed in amber glass flasks containing the filtered aqueous samples $(100 \mathrm{~mL}), 500 \mu \mathrm{L}$ of acetic anhydride, $10 \mathrm{~g} \mathrm{~L}^{-1}$ of sodium carbonate and $100 \mathrm{~g} \mathrm{~L}^{-1}$ of sodium chloride, and stirred at $900 \mathrm{rpm}$ during $5 \mathrm{~h}$ at room temperature.

After extraction, the PDMS bars were gently dried, and analytes were desorbed by liquid desorption (LD). PDMS bars were sonicated during $30 \mathrm{~min}$ in $2 \mathrm{~mL}$ vials filled with inserts containing $200 \mu \mathrm{L}$ of ethyl acetate, this condition optimized based on a previous publication [16]. $1 \mu \mathrm{L}$ of sample was then injected into the GC system. After use, all stir bars were cleaned during $24 \mathrm{~h}$ using a mixture of DCM and $\mathrm{MeOH}(1: 1, v / v)$, and later, sonicated during 30 min using the same solvent mixture [22].

\subsection{Instruments}

Capillary gas chromatography separation of analytes was performed on an Agilent 7890N using a HP-5MS column $(30 \mathrm{~m}$ $\times 0.25 \mathrm{~mm}$ i.d. $\times 0.25 \mu \mathrm{m}$ film thickness consisting of $5 \%$ phenyl and $95 \%$ polydimethylsiloxane), keeping the helium carrier gas flow at $1 \mathrm{~mL} \mathrm{~min}{ }^{-1}$ and the injection port temperature at $280^{\circ} \mathrm{C}$. The column temperature ramp was as follows: $70^{\circ} \mathrm{C}$ for $1 \mathrm{~min}$, increased by $35^{\circ} \mathrm{C} \mathrm{min}^{-1}$ to $180^{\circ} \mathrm{C}$, then by $4.50^{\circ} \mathrm{C} \mathrm{min}^{-1}$ to $290^{\circ} \mathrm{C}$, and held for $8 \mathrm{~min}$.

Time-of-flight mass spectrometry was used for the identification and quantification of analytes (Waters Synapt G2). APGC ionization was performed by alternating high and low energies within the same run, thus obtaining - within the same analysis molecular and fragment ions for target (and non-target) compounds. The mass range considered was $m / z=50-600$. Corona voltage was $2 \mathrm{kV}$, and the source temperature was $130^{\circ} \mathrm{C}$. Different sampling cone voltages (from 10 to $40 \mathrm{~V}$ ) were tested. Identification of analytes was based on comparing retention times and accurate mass measurements (allowing an error of less than $5 \mathrm{ppm}$ ) to those for commercially available pure standards. Additionally, selected standards and environmental samples were re-analyzed using EI $(70 \mathrm{eV})$ instead of APGC.

Quantification of target compounds was performed using calibration curves (from 1 to $500 \mu \mathrm{g} \mathrm{L}^{-1}$, prepared in ethyl acetate in $2 \mathrm{~mL}$ vials, and from 0.001 to $1 \mu \mathrm{g} \mathrm{L}^{-1}$, prepared in HPLC water in $100 \mathrm{~mL}$ flasks that underwent SBSE) and taking into account the signal intensities of the surrogates. The reproducibility and repeatability of the methods were evaluated by performing three successive extractions and injections of the same sample and by re-analyzing a batch of standards two weeks after its first analysis. The lowest concentrations at which the signal-to-noise ratio was greater than 3 in a standard solution and in a spiked HPLC water sample extracted by SBSE were considered to be the instrumental (IDL) and method detection limits (MDL), respectively.

\section{Results and discussion}

\subsection{Extraction of target compounds}

During the development of this multiresidue method, and due to the large number and diversity of analytes considered, several key parameters affecting the extraction efficiency of 
SBSE were evaluated and optimized by means of several experiments. These parameters were: type and volume of the derivatization agent, PDMS bar size, sample volume, agitation time, ionic strength $(\% \mathrm{NaCl}), \mathrm{pH}$, and amount of organic modifier (\% methanol added to the sample). All the experiments were performed at room temperature $\left(20^{\circ} \mathrm{C}\right)$ and in darkness to avoid possible photodegradation of light-sensitive compounds. Taking into account available information on SBSE method development $[15,16]$, the following parameters were fixed at the start to reduce the number of experiments: room temperature, agitation time $(12 \mathrm{~h})$, agitation speed $(900 \mathrm{rpm})$, stir bar size $(10 \mathrm{~mm})$, and neutral $\mathrm{pH}$.

\subsubsection{Derivatizing agent}

The first set of experiments was undertaken to decide whether to use derivatizing agents. Fig. 1A shows the results from adding different volumes of acetic acid anhydride (10, 50, 100 and $200 \mu \mathrm{L}$ ) to $10 \mathrm{~mL}$ HPLC water aliquots spiked with target compounds. This agent reacts with hydroxyl and phenol groups in target compounds such as TCS, BpA or HMS $[23,24]$, therefore reducing their polarity and improving volatility, sensitivity and chromatographic separation. Other agents such as $\quad N$-methyl- $N$-(tert-butyldimethylsilyl) trifluoroacetamide (MTBSTFA) were also considered but the silylation reaction does not take place in water [25], whereas acetic acid anhydride allows in situ derivatization [24-26]. Acetylation of selected analytes also allows achieving better extraction recovery percentages and detection limits in the method (MDLs) (e.g., E1) than using other derivatizing agents such as MTBSTFA or $\mathrm{N}, \mathrm{O}$-bis(trimethylsilyl)trifluoroacetamide (BSTFA) and $1 \%$ trimethylchlorosilane (TMCS) [27], as the compounds formed have higher affinity for the PDMS bars. Most of the regulated compounds did not require this in situ derivatization step, but signal intensities increased noticeably for some emerging contaminants such as E1, E2, EE, TCS, BpA, 3-OHBP, and OP, among others (Fig. 1A). Recoveries for those compounds not reacting with acetic acid anhydride remained unaffected in most cases (e.g., 4-MBC, 4,4-DDE, and MK), although variations up to $20 \%$ where observed occasionally (e.g., Musk R1). The signal of those compounds undergoing derivatization increased by $20-30 \%$ when the volume of reagent had been increased from 10 to $50 \mu \mathrm{L}$. In general, adding a higher amount of acetic acid anhydride $(100-200 \mu \mathrm{L})$ did not provide significantly better results (with the notable exception of nonylphenol) and extraction of some of the compounds that do not require derivatization was lower (e.g, dibenzo[a]anthracene, propazine). As a result, we decided to choose $50 \mu \mathrm{L}$ as a compromise (500 $\mu \mathrm{L}$ for $100 \mathrm{~mL}$ samples).

The addition of acetic acid anhydride often leads to a decrease in the $\mathrm{pH}$ of the aqueous samples $(\mathrm{pH} 3)$. Therefore, this effect was also investigated by adjusting the $\mathrm{pH}$ adding different amounts of sodium carbonate: $0 \mathrm{gL}^{-1}(\mathrm{pH} 3), 10 \mathrm{gL}^{-1}(\mathrm{pH} 7)$ and $50 \mathrm{gL}^{-1}$

A

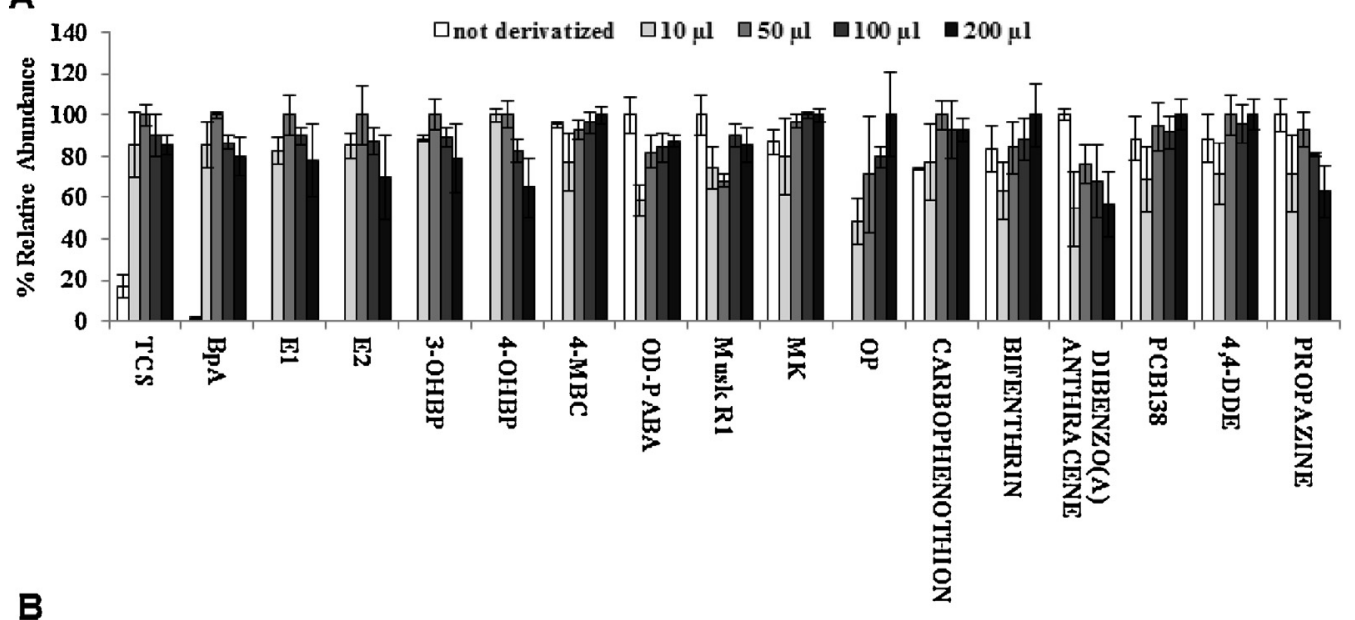

B

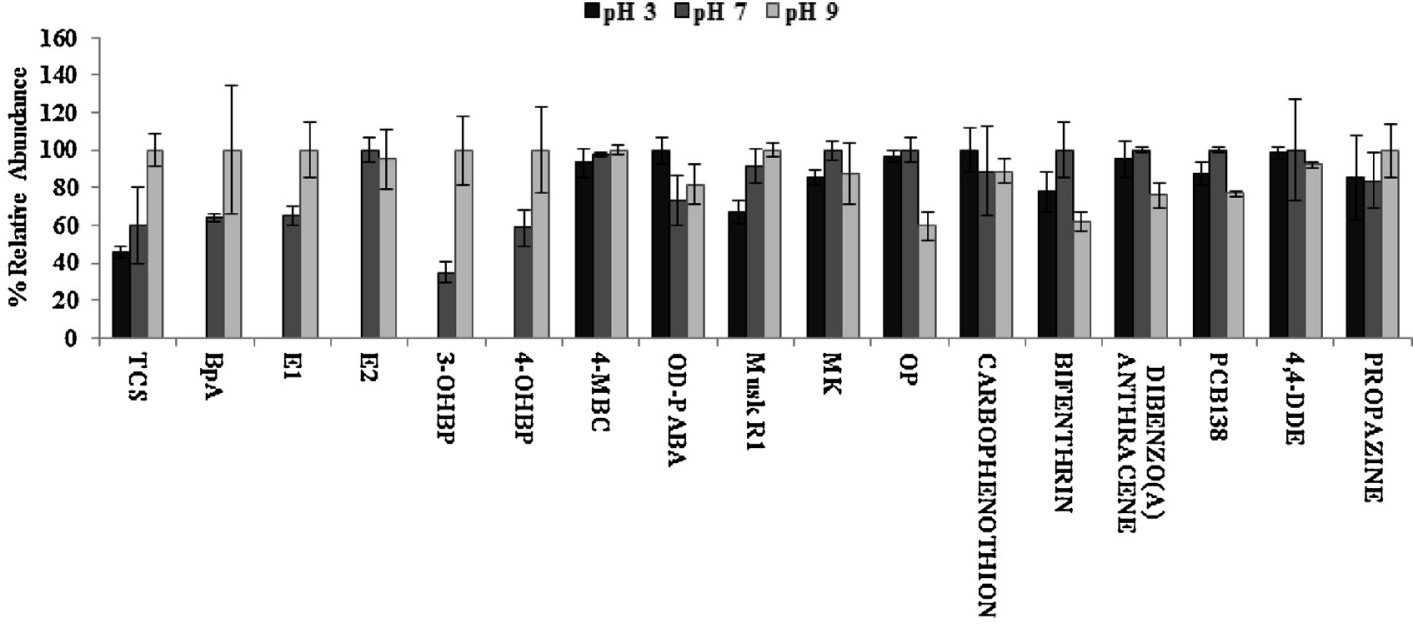

Fig. 1. Influence of the derivatization agent volume $(\mathrm{A})$ and $\mathrm{pH}(\mathrm{B})$ on the extraction of selected target compounds by SBSE. 


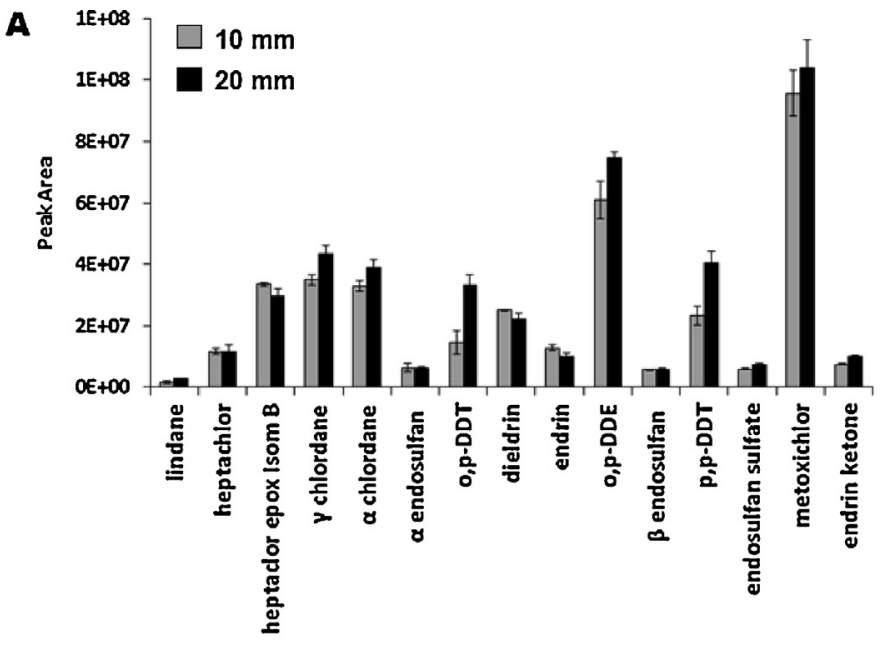

$\mathbf{B}$

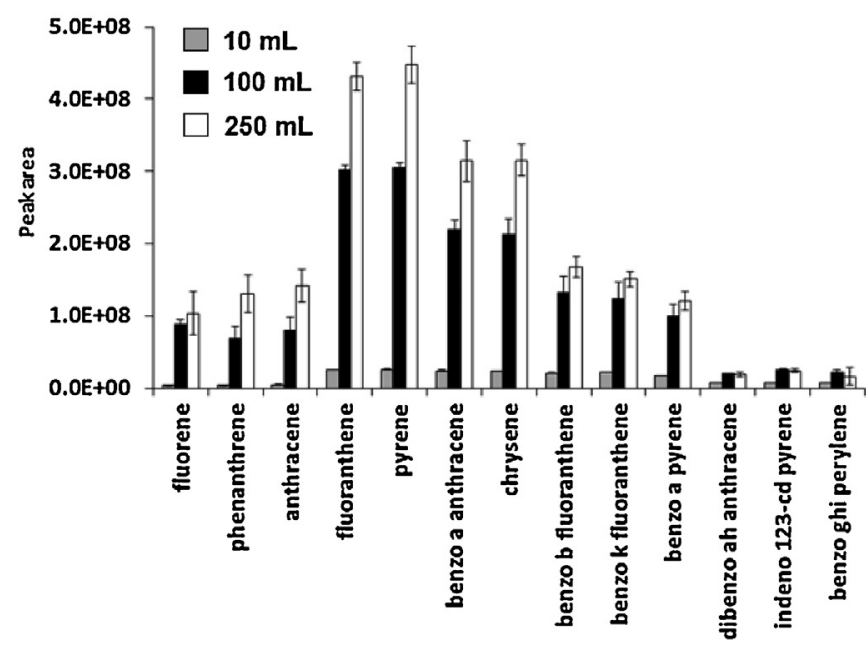

Fig. 2. Influence of the PDMS bar size (A) and sample volume (B) on the extraction of selected target compounds by SBSE.

( $\mathrm{pH}$ 9). Low $\mathrm{pH}$ values enhance the protonation of many target compounds, decreasing the reaction yield with the derivatizing agent, acetic anhydride. Thus, we can observe in Fig. 1B that many of the compounds that rely on derivatization to be analyzed $(\mathrm{BpA}$, $\mathrm{E} 1, \mathrm{E} 2$, etc.) were barely detected at $\mathrm{pH} 3$. Higher signal intensities were obtained at basic $\mathrm{pH}$ values although we could also observe a decrease in the extraction efficiency of other target compounds (e.g. PCBs and pyrethroids) $[14,28]$. Finally, neutral $\mathrm{pH}$ was selected as a compromise to perform further SBSE experiments.

\subsubsection{Other SBSE parameters}

Sample volume $(10,100$ or $200 \mathrm{~mL})$ and stir bar size $(10 \mathrm{~mm}$ $\times 0.5 \mathrm{~mm}$ or $20 \mathrm{~mm} \times 0.5 \mathrm{~mm}$ ) were tested next. The size bar selected was $10 \mathrm{~mm}$ because only small differences in the signal intensity (less than $15 \%$ in most cases) compared to $20 \mathrm{~mm}$ were observed and, most important, the amount of solvent needed during the liquid desorption step was reduced (see Fig. 2A). Regarding the effect of the sample volume, Fig. 2B shows signal intensities of different PAHs for several sample volumes $(10,100$, and $250 \mathrm{~mL}$ ). A 10 -fold increase in the signal intensity was measured for most compounds when moving from 10 to $100 \mathrm{~mL}$ samples. Moving towards higher volumes $(250 \mathrm{~mL})$, the effect of sample volume is more noticeable for more polar compounds (e.g., fluorene, $\log K_{\mathrm{ow}}=4.02$ ), whereas differences were lower than $10-20 \%$ for more hydrophobic PAHs (e.g., dibenzo[ $a, h]$ anthracene, $\log K_{\text {ow }}=6.7$ ). In any case, and although using $250 \mathrm{~mL}$ led to further signal increase, it proved unnecessary taking into account the low detection limits obtained for $100 \mathrm{~mL}$ experiments (Table 1 ). This sample volume was considered to be enough for monitoring target compounds in the environment at ppt levels, which is in agreement with previous findings [15,29]. If sample volume is not a limiting factor, and the chemicals are expected to be at very low concentrations (i.e. open ocean water, groundwater), using $250 \mathrm{~mL}$ instead of $100 \mathrm{~mL}$ would be recommended.

The effects of ionic strength $\left(0,100\right.$, and $200 \mathrm{~g} \mathrm{~L}^{-1}$ of $\left.\mathrm{NaCl}\right)$ and presence of organic modifiers $(0,10$, and $20 \%$ of methanol) in the samples were studied next. Fig. 3 shows signal intensities for selected compounds at different conditions. Presence of salt was mandatory for some compounds such as triazines (e.g., terbuthylazine) where $200 \mathrm{~g} \mathrm{~L}^{-1}$ increased recoveries by more than $40 \%$. Adding salt also improved the extraction of many polar compounds $\left(\log K_{\mathrm{ow}}<4\right)$, mainly those having hydroxyl groups their structure (e.g., E1, E2, EE, OP, 3-OHBP, and BP-3), insect repellents like DEET, and some pesticides (parathion, endrin ketone, and lindane). This benefit, however, was offset by the large losses generated in more hydrophobic compounds (e.g. galaxolide, EHMC, helvetolide, cyfluyhrins, musk ambrette, benzo[g,h,i]perylene, etc.) [30]. This was more noticeable when the highest amount of salt $\left(200 \mathrm{~g} \mathrm{~L}^{-1}\right)$ was added, especially for those non-polar compounds showing highest molecular weights $\left(\log K_{\mathrm{ow}}>6\right)$ such as PAHs with a high

Table 1

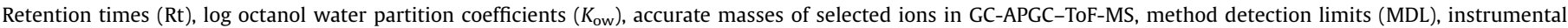
detection limits (IDL), calibration curves, and coefficients of determination $\left(R^{2}\right)$ for target compounds.

\begin{tabular}{|c|c|c|c|c|c|c|c|}
\hline Target compound & $\log K_{\mathrm{ow}}$ & $\mathrm{Rt}(\min )$ & Accurate mass (Da) & $\operatorname{MDL}\left(\mathrm{ng} \mathrm{L}^{-1}\right)$ & $\operatorname{IDL}\left(\mu \mathrm{g} \mathrm{L}^{-1}\right)$ & Calibration curve & $R^{2}$ \\
\hline \multicolumn{8}{|l|}{ Polycyclic musks } \\
\hline Galaxolide & $5.9^{\mathrm{a}}$ & 8.95 & $259.206 / 189.128$ & 0.04 & 0.019 & $y=110.925 x+1.826$ & 0.9944 \\
\hline Tonalide & $5.7^{\mathrm{a}}$ & 9.1 & 259.2062 & 0.02 & 0.012 & $y=176.821 x+6.191$ & 0.9929 \\
\hline Traseolide & $5.76^{c}$ & 8.9 & 215.144 & 0.22 & 0.107 & $y=19.3486 x+0.240$ & 0.9753 \\
\hline Phantolide & $5.3^{c}$ & 7.9 & 245.1905 & 0.04 & 0.009 & $y=205.871 x-0.0322$ & 0.9949 \\
\hline Celestolide & $5.93^{\mathrm{c}}$ & 7.47 & 245.1905 & 0.09 & 0.010 & $y=180.696 x-0.217$ & 0.9983 \\
\hline \multicolumn{8}{|l|}{ Nitro musks } \\
\hline Musk xylene & $4.4^{\mathrm{c}}$ & 9.08 & 298.096 & 0.02 & 3.594 & $y=9.61756 x+0.187$ & 0.9983 \\
\hline Musk ambrette & $5.7^{\mathrm{a}}$ & 8.75 & 269.1137 & 0.03 & 0.049 & $y=219.037 x+1.139$ & 0.9984 \\
\hline Musk tibetene & $5.9^{\mathrm{a}}$ & 10.09 & 251.1039 & 0.05 & 0.048 & $y=47.286 x+1.166$ & 0.9906 \\
\hline Musk ketone & $4.8^{\mathrm{a}}$ & 10.65 & 279.0981 & 0.95 & 0.227 & $y=16.0167 x+0.601$ & 0.9892 \\
\hline Musk moskene & $5.8^{\mathrm{a}}$ & 9.4 & 279.135 & 0.02 & 0.015 & $y=192.425 x+1.654$ & 0.9963 \\
\hline \multicolumn{8}{|l|}{ Macrocyclic musks } \\
\hline Muscone & $5.96^{\mathrm{c}}$ & 8.98 & 239.2375 & 0.12 & 0.031 & $y=67.4382 x+0.816$ & 0.9987 \\
\hline
\end{tabular}


Table1 (Continued)

\begin{tabular}{|c|c|c|c|c|c|c|c|}
\hline Target compound & $\log K_{\mathrm{ow}}$ & Rt (min) & Accurate mass (Da) & $\operatorname{MDL}\left(\mathrm{ng} \mathrm{L}^{-1}\right)$ & $\operatorname{IDL}\left(\mu \mathrm{g} \mathrm{L}^{-1}\right)$ & Calibration curve & $R^{2}$ \\
\hline Musk R1 & $6.65^{c}$ & 9.74 & 257.2124 & 0.43 & 0.055 & $y=97.6912 x-0.651$ & 0.997 \\
\hline Habanolide & $4.88^{\mathrm{c}}$ & 8.64 & 239.2011 & 1.4 & 0.076 & $y=15.3471 x+0.064$ & 0.9852 \\
\hline Exaltenone & $6.15^{\mathrm{c}}$ & 8.59 & 223.2062 & 0.79 & 0.079 & $y=147.111 x-1.641$ & 0.9966 \\
\hline Muscenone & & 9.04 & 237.2218 & 0.73 & 0.122 & $y=30.6426 x+0.607$ & 0.9094 \\
\hline \multicolumn{8}{|l|}{ Other fragrances } \\
\hline Helvetolide & $5.51^{\mathrm{c}}$ & 7.38 & 129.092 & 0.59 & 0.455 & $y=12.2424 x-0.043$ & 0.9991 \\
\hline Cashmeran & $4.9^{\mathrm{a}}$ & 5.79 & 207.1749 & 0.2 & 0.078 & $y=138.101 x-2.042$ & 0.9955 \\
\hline OTNE & $5.6^{c}$ & 7.04 & 235.2062 & 0.2 & 0.211 & $y=13.9104 x+1.702$ & 0.9835 \\
\hline \multicolumn{8}{|l|}{ Insect repellents } \\
\hline IRGAROL & $4.07^{\mathrm{c}}$ & 11.92 & 254.1439 & 0.03 & 0.019 & $y=6.8686 x-0.088$ & 0.9899 \\
\hline DEET & $2.26^{c}$ & 6.25 & 192.1388 & 0.35 & 0.038 & $y=6.7033 x+0.0368$ & 0.9989 \\
\hline \multicolumn{8}{|l|}{ Antibacterials } \\
\hline TCS & $4.66^{\mathrm{c}}$ & 13.55 & 289.951 & 0.04 & 0.074 & $y=66.2642 x-0.383$ & 0.9843 \\
\hline MTCS & $5.2^{\mathrm{c}}$ & 12.74 & 302.9746 & 0.04 & 0.024 & $y=16.2516 x-0.027$ & 0.9825 \\
\hline \multicolumn{8}{|c|}{ Endocrine disrupting compounds } \\
\hline NP & $5.99^{c}$ & 10.3 & 221.1907 & 0.71 & 1.953 & $y=161.328 x-7.844$ & 0.9675 \\
\hline $\mathrm{OP}$ & $5.5^{\mathrm{c}}$ & 8.96 & 207.1757 & 0.22 & 1.508 & $y=284.781 x-14.927$ & 0.9579 \\
\hline BpA & $3.64^{\mathrm{c}}$ & 16.47 & 228.115 & 0.03 & 1.573 & $y=35.8926 x+0.133$ & 0.9889 \\
\hline E1 & $3.43^{c}$ & 22.4 & 271.1698 & 0.3 & 2.046 & $y=47.1716 x-0.199$ & 0.9953 \\
\hline E2 & $3.94^{\mathrm{c}}$ & 22.7 & 273.1855 & 0.73 & 2.006 & $y=15.2828 x-0.113$ & 0.9897 \\
\hline EE2 & $4.12^{\mathrm{c}}$ & 23.8 & 297.1855 & 1.37 & 3.134 & $y=17.5645 x-0.075$ & 0.9937 \\
\hline \multicolumn{8}{|l|}{ UV-filters } \\
\hline $\mathrm{BzS}$ & $4.31^{\mathrm{c}}$ & 9.15 & 91.0561 & 2.15 & 21.082 & $y=0.24369 x+0.829$ & 0.9402 \\
\hline EHS & $4.86^{\mathrm{c}}$ & 8.33 & 139.0405 & 0.28 & 0.329 & $y=9.659085 x+0.655$ & 0.9482 \\
\hline HMS & $6.16^{\mathrm{c}}$ & 9.35 & 139.0402 & 0.44 & 0.335 & $y=9.13551 x+0.446$ & 0.9609 \\
\hline $4-\mathrm{MBC}$ & $5.92^{\mathrm{c}}$ & 11.74 & 255.1749 & 0.01 & 0.020 & $y=1.25678 x-20.577$ & 0.9763 \\
\hline OC & $6.8^{\mathrm{c}}$ & 21.41 & 250.087 & 0.02 & 0.029 & $y=83.9559 x+9.762$ & 0.9238 \\
\hline EHMC & $5.8^{\mathrm{c}}$ & 15.69 & 179.0713 & 0.46 & 0.367 & $y=66.852 x+2.087$ & 0.9738 \\
\hline OD-PABA & $5.69^{c}$ & 14.91 & 166.087 & 0.6 & 0.135 & $y=56.9226 x-0.365$ & 0.969 \\
\hline 2-OHBP & $3.44^{\mathrm{c}}$ & 7.62 & 199.0759 & 0.53 & 1.142 & $y=3.3361 x+0.208$ & 0.9632 \\
\hline Avobenzone & $4.51^{\mathrm{c}}$ & 22.4 & 311.165 & 12.4 & 28.986 & $y=1.48623 x+0.011$ & 0.9599 \\
\hline 3-OHBP & 3.44 & 9.22 & 199.0759 & 0.61 & 0.857 & $y=17.7044 x-0.173$ & 0.9505 \\
\hline 4-OHBP & 3.44 & 11.33 & 199.0759 & 1.55 & 0.857 & $y=16.5988 x-0.068$ & 0.9015 \\
\hline BP-3 & $3.52^{\mathrm{c}}$ & 13.15 & 229.0865 & 0.17 & 0.921 & $y=0.914866 x-1.397$ & 0.9807 \\
\hline BP-10 & $4.07^{\mathrm{c}}$ & 13.25 & 243.1021 & 1.66 & 0.393 & $y=10.3146 x-0.234$ & 0.906 \\
\hline \multicolumn{8}{|l|}{ PAHs } \\
\hline Acenaphthylene & $3.17^{\mathrm{b}}$ & 5.53 & 153.0704 & 0.68 & 0.209 & $y=2.43077 x+0.021$ & 0.9889 \\
\hline Acenaphthene & $4.15^{\mathrm{c}}$ & 5.78 & 155.0861 & 1.02 & 0.161 & $y=11.5878 x-0.053$ & 0.9727 \\
\hline Fluorene & $4.02^{\mathrm{b}}$ & 6.43 & 167.0861 & 0.26 & 0.112 & $y=13.4396 x+0.016$ & 0.9706 \\
\hline Phenanthrene & $4.35^{\mathrm{b}}$ & 8.28 & 179.0861 & 0.22 & 0.099 & $y=10.3956 x+0.081$ & 0.9528 \\
\hline Anthracene & $4.35^{\mathrm{b}}$ & 8.36 & 179.0861 & 0.67 & 0.158 & $y=13.1884 x+0.051$ & 0.9633 \\
\hline Fluoranthene & $4.93^{\mathrm{b}}$ & 11.85 & 203.0861 & 0.12 & 0.193 & $y=20.2895 x+0.165$ & 0.9281 \\
\hline Pyrene & $4.93^{\mathrm{b}}$ & 12.64 & 203.0861 & 0.1 & 0.220 & $y=17.1829 x+0.162$ & 0.9196 \\
\hline Benzo[a]anthracene & $5.52^{\mathrm{b}}$ & 17.84 & 229.1017 & 0.26 & 0.246 & $y=7.56888 x+0.074$ & 0.9125 \\
\hline Chrysene & $5.52^{\mathrm{b}}$ & 18.02 & 229.1017 & 0.16 & 0.122 & $y=9.2828 x+0.123$ & 0.9181 \\
\hline Benzo[b]fluoranthene & $6.11^{\mathrm{b}}$ & 23.78 & 253.1017 & 0.37 & 0.280 & $y=18.222 x+0.701$ & 0.9361 \\
\hline Benzo[k]fluoranthene & $6.11^{\mathrm{b}}$ & 22.89 & 253.1017 & 1.02 & 0.690 & $y=19.9449 x+0.818$ & 0.9049 \\
\hline Benzo[a]pyrene & $6.11^{\mathrm{b}}$ & 24.11 & 253.1017 & 1.24 & 0.815 & $y=11.7329 x+0.753$ & 0.913 \\
\hline Dibenzo[ $a, h]$ anthracene & $6.7^{\mathrm{b}}$ & 28.86 & 279.1174 & 5.97 & 1.407 & $y=2.8057 x+0.161$ & 0.9426 \\
\hline Indeno[1,2,3-cd]pyrene & $6.7^{\mathrm{b}}$ & 28.65 & 277.1017 & 5.13 & 1.078 & $y=3.28973 x+0.216$ & 0.9732 \\
\hline Benzo[g,h,i]perylene & $6.7^{\mathrm{b}}$ & 29.56 & 277.1017 & 4.65 & 0.837 & $y=3.85966 x+0.328$ & 0.9581 \\
\hline \multicolumn{8}{|l|}{ PCBs } \\
\hline PCB 52 & $5.79^{\mathrm{b}}$ & 10.14 & 289.9224 & 0.05 & 0.021 & $y=6.11665 x+0.103$ & 0.9567 \\
\hline PCB 28 & $5.71^{\mathrm{b}}$ & 9.24 & 255.9613 & 0.07 & 0.144 & $y=29.8702 x+0.361$ & 0.9376 \\
\hline PCB 138 & $6.82^{\mathrm{b}}$ & 15.45 & 357.8444 & 0.12 & 0.031 & $y=0.55267 x+0.002$ & 0.9027 \\
\hline PCB 153 & $7.29^{\mathrm{b}}$ & 16.42 & 357.8444 & 0.49 & 0.028 & $y=0.95481 x+0.004$ & 0.9001 \\
\hline РСВ 180 & $7.21^{\mathrm{b}}$ & 18.83 & 391.8054 & 0.18 & 0.249 & $y=0.20016 x+0.008$ & 0.9411 \\
\hline РCВ 101 & $6.98^{\mathrm{c}}$ & 12.65 & 323.8834 & 0.01 & 0.004 & $y=0.52695 x+0.009$ & 0.9521 \\
\hline \multicolumn{8}{|l|}{ Organochlorine pesticides } \\
\hline Lindane & $4.14^{\mathrm{b}}$ & 7.38 & 129.092 & 0.43 & 0.455 & $y=8.69311 x+0.002$ & 0.9838 \\
\hline Heptachlor & $5.47^{\mathrm{b}}$ & 9.64 & 336.849 & 0.02 & 0.003 & $y=3.55588 x+0.175$ & 0.9547 \\
\hline Heptaclor epoxide IsomB & $4.98^{\mathrm{b}}$ & 11.71 & 352.846 & 0.01 & 0.039 & $y=1.76856 x+0.021$ & 0.9325 \\
\hline$\alpha$ Chlordane & $6.16^{\mathrm{c}}$ & 12.43 & 372.8253 & 0.18 & 0.052 & $y=0.692043 x+0.002$ & 0.9591 \\
\hline$\gamma$ Chlordane & $6.16^{\mathrm{c}}$ & 12.9 & 372.8253 & 0.44 & 0.091 & $y=0.391388 x+0.028$ & 0.907 \\
\hline$\alpha$ Endosulfan & $3.83^{\mathrm{b}}$ & 12.8 & 404.8247 & 0.06 & 0.004 & $y=1.58856 x+0.017$ & 0.9569 \\
\hline$o, p^{\prime}$-DDT & $6.91^{\mathrm{b}}$ & 14.98 & 235.0083 & 0.15 & 0.101 & $y=1.29556 x+0.008$ & 0.9008 \\
\hline Dieldrin & $5.4^{\mathrm{b}}$ & 13.64 & 380.875 & 0.03 & 0.024 & $y=2.85297 x+0.036$ & 0.9277 \\
\hline Endrin & $5.2^{\mathrm{b}}$ & 14.34 & 378.8785 & 0.09 & 0.286 & $y=1.53037 x+0.030$ & 0.9072 \\
\hline$o, p^{\prime}-\mathrm{DDE}$ & $6.02^{\mathrm{b}}$ & 13.58 & 317.935 & 0.02 & 0.004 & $y=0.387684 x+0.015$ & 0.9172 \\
\hline
\end{tabular}


Table1 (Continued)

\begin{tabular}{|c|c|c|c|c|c|c|c|}
\hline Target compound & $\log K_{\text {ow }}$ & Rt (min) & Accurate mass (Da) & $\operatorname{MDL}\left(\mathrm{ng} \mathrm{L}^{-1}\right)$ & $\operatorname{IDL}\left(\mu \mathrm{g} \mathrm{L}^{-1}\right)$ & Calibration curve & $R^{2}$ \\
\hline$\beta$ Endosulfan & $3.83^{b}$ & 14.64 & 404.8247 & 0.03 & 0.006 & $y=0.527011 x+0.029$ & 0.952 \\
\hline$p, p^{\prime}-\mathrm{DDT}$ & $6.91^{\mathrm{b}}$ & 16.28 & 235.0081 & 1.67 & 0.270 & $y=0.312321 x+0.005$ & 0.9342 \\
\hline Endosulfan sulfate & $3.66^{\mathrm{b}}$ & 16.1 & 324.849 & 0.01 & 0.040 & $y=1.85753 x+0.012$ & 0.9823 \\
\hline Metoxychlor & $5.08^{\mathrm{b}}$ & 18.39 & 345.0216 & 3.08 & 3.133 & $y=0.273402 x+0.005$ & 0.9517 \\
\hline Endrin ketone & $4.99^{\mathrm{b}}$ & 17.73 & 378.8781 & 0.16 & 0.122 & $y=1.33013 x+0.004$ & 0.9754 \\
\hline \multicolumn{8}{|c|}{ Organophosphate pesticides } \\
\hline Parathion & $3.73^{\mathrm{b}}$ & 10.75 & 292.0409 & 0.03 & 0.009 & $y=19.7751 x+0.101$ & 0.9218 \\
\hline Ethion & $4.75^{c}$ & 15.21 & 384.9954 & 0.1 & 0.007 & $y=3.742 x+0.036$ & 0.9347 \\
\hline Carbophenothion & $5.19^{c}$ & 10.58 & 331.8956 & 0.29 & 0.058 & $y=0.2,542 x+0.0004$ & 0.9805 \\
\hline Chlorpyrifos & $4.66^{c}$ & 10.72 & 349.933 & 0.03 & 0.007 & $y=35.8774 x+0.392$ & 0.972 \\
\hline \multicolumn{8}{|l|}{ Triazines } \\
\hline Ametryn & $3.32^{\mathrm{b}}$ & 9.6 & 228.1283 & 0.39 & 0.030 & $y=20.8727 x+0.146$ & 0.9181 \\
\hline Atrazine & $2.82^{\mathrm{b}}$ & 7.72 & 216.1016 & 1.21 & 0.012 & $y=2.24777 x+0.005$ & 0.9919 \\
\hline Prometon & $3.57^{\mathrm{b}}$ & 7.62 & 226.1668 & 0.3 & 0.116 & $y=15.9175 x+0.159$ & 0.9302 \\
\hline Secbumeton & $3.64^{c}$ & 8.41 & 226.1668 & 2.51 & 0.243 & $y=10.2501 x+0.121$ & 0.9217 \\
\hline Prometryn & $3.73^{\mathrm{b}}$ & 9.69 & 242.1439 & 0.11 & 0.005 & $y=56.0515 x+0.159$ & 0.9214 \\
\hline Terbutryn & $3.77^{\mathrm{b}}$ & 10.04 & 242.1439 & 0.13 & 0.012 & $y=42.8802 x+0.141$ & 0.9273 \\
\hline Propazine & $3.24^{b}$ & 7.81 & 230.1172 & 1.31 & 0.042 & $y=8.89159 x+0.165$ & 0.9038 \\
\hline Terbuthylazine & $3.27^{\mathrm{b}}$ & 8.03 & 230.1172 & 2.08 & 0.120 & $y=14.7927 x+0.160$ & 0.9298 \\
\hline Simetryn & $2.90^{\mathrm{c}}$ & 9.5 & 214.1126 & 0.83 & 0.037 & $y=5.93133 x+0.130$ & 0.9007 \\
\hline Simazine & $2.40^{c}$ & 7.63 & 202.0859 & 17.73 & 0.088 & $y=0.701433 x-0.005$ & 0.9798 \\
\hline \multicolumn{8}{|l|}{ Pyrethroids } \\
\hline Bifenthrin & $8.15^{c}$ & 18.34 & 181.1028 & 1.82 & 0.104 & $y=1.1511 x+0.0201$ & 0.9684 \\
\hline Phenothrin I & $6.1^{\mathrm{c}}$ & 19.08 & 351.1952 & 0.4 & 2.489 & $y=0.692254 x+0.187$ & 0.9073 \\
\hline Phenothrin II & $6.1^{\mathrm{c}}$ & 19.3 & 351.1952 & 0.78 & 2.351 & $y=0.912941 x+0.268$ & 0.907 \\
\hline Permethrin I & $6.18^{\mathrm{c}}$ & 21.82 & 183.0817 & 2.3 & 1.037 & $y=0.434399 x+0.049$ & 0.9631 \\
\hline Permethrin II & $6.18^{\mathrm{c}}$ & 22.08 & 355.0698 & 4.94 & 0.980 & $y=0.67445 x+0.145$ & 0.9332 \\
\hline Cyfluthrins (I-IV) & $5.9^{c}$ & $23.1-23.6$ & 191.0037 & 2.2 & 2.271 & $y=1.21931 x+0.089$ & 0.9101 \\
\hline Cypermethrin (I-IV) & $6.38^{c}$ & $23.7-24.2$ & 191.0037 & 2.12 & 4.289 & $y=1.11045 x+0.082$ & 0.9155 \\
\hline Deltamethrin I & $6.18^{\mathrm{c}}$ & 26.8 & 280.9006 & 5.52 & 1.727 & $y=0.306606 x+0.005$ & 0.9091 \\
\hline Deltamethrin II & $6.18^{\mathrm{c}}$ & 27.23 & 280.9006 & 1.63 & 0.156 & $y=0.287318 x+0.009$ & 0.9601 \\
\hline Fenvalerate I & $6.76^{\mathrm{c}}$ & 25.65 & 167.0628 & 3.88 & 1.221 & $y=0.0131 x-0.061$ & 0.9947 \\
\hline Fenvalerate II & $6.76^{c}$ & 26.11 & 167.0628 & 7.36 & 2.295 & $y=0.007 x-0.037$ & 0.9952 \\
\hline \multicolumn{8}{|l|}{ Surrogates } \\
\hline Terbuthylazine d5 & & 7.93 & 235.1497 & & & & \\
\hline Chrysene d12 & & 17.95 & 241.1769 & & & & \\
\hline Perylene d12 & & 24.42 & 265.1765 & & & & \\
\hline Phenanthrene d10 & & 8.29 & 189.1285 & & & & \\
\hline Musk xylene d15 & & 8.91 & 294.1481 & & & & \\
\hline Triclosan d3 & & 13.5 & 292.9671 & & & & \\
\hline Methyl triclosan ${ }^{13} \mathrm{C}_{12}$ & & 12.71 & 306.9838 & & & & \\
\hline Benzophenone $\mathrm{d} 10$ & & 6.69 & 188.1128 & & & & \\
\hline
\end{tabular}

${ }^{a}$ Octanol-water coefficients obtained from SCIFINDER

b Octanol-water coefficients are obtained from [15].

c Octanol-water coefficients are obtained from KOWWIN.

number of rings (e.g., dibenzo[ $a, h]$ anthracene), PCBs with many chlorine atoms (e.g., PCB 138), pyrethroids, and some UV-filters, all of them showing higher recovery percentages in the absence of $\mathrm{NaCl}$. This effect could be partly counteracted by the addition of an organic solvent (methanol), which was also intended to minimize the possible adsorption of more hydrophobic compounds onto the glass walls of the flasks [1,16]. Thus, a positive response was observed for some compounds such as metoxichlor, 4,4-DDD, carbophenothion and musk ketone, although the effect was not dramatic $(<30 \%)$ as most of these chemicals already have high affinity for the stir bars rather than for the glass walls. The addition of methanol (10-20\%) could also compensate the negative effect when salt was added (e.g., see PCB 138 and dibenzo[ $a, h]$ anthracene in Fig. 3). However, heavy losses were detected for many compounds when the amount of methanol reached 20\%, presumably due to a decrease in their interaction with the PDMS bar (e.g., E1, terbuthylazine). Considering all data available, we decided to add only $100 \mathrm{~g} \mathrm{~L}^{-1}$ of $\mathrm{NaCl}$ and no methanol to aqueous samples to enhance the extraction of those analytes having lower $K_{\text {ow }}$ coefficients (e.g., E2, DEET, 2-OHBP, 3-OHBP, and triazines), which are often more problematic than hydrophobic compounds when SBSE is performed [31- 32]. Seawater salinity $\left(35 \mathrm{gL}^{-1}\right)$ was considered when adding the proper amount of $\mathrm{NaCl}$ to samples of marine origin. An alternative approach could be using a sequential SBSE method, previously used by other authors [33,34], and consisting on changing the conditions of the extraction over time to enhance the extraction of a wide range of analytes. However, we decided to perform a single extraction step under stable conditions due to the addition of a derivatizing agent.

Finally, another set of experiments aimed to optimize agitation time was performed. The objective was to determine the time necessary for the compounds to reach the equilibrium between the PDMS bar and the aqueous solution. Extracting analytes at this time is critical to achieve the highest accuracy and sensitivity [14]. Agitation time was optimized by stirring the samples at different time periods $(0,0.5,1,2,3,4,6,8$ and $24 \mathrm{~h})$. Results for some selected compounds are shown in Fig. 4. Equilibrium conditions were reached between 4 and $5 \mathrm{~h}$ for most compounds, and no significant changes in the extraction efficiency could be observed afterwards. Most authors use extraction periods of 4-6h for environmental analysis of aqueous samples [29,35]. Under 
Pantholide

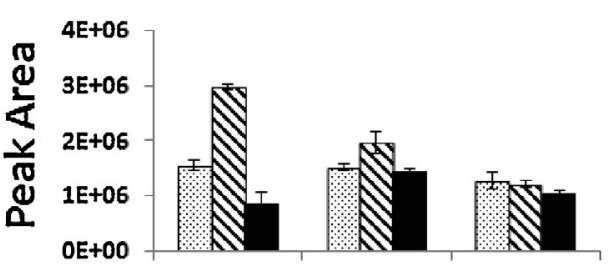

Musk Ambrette

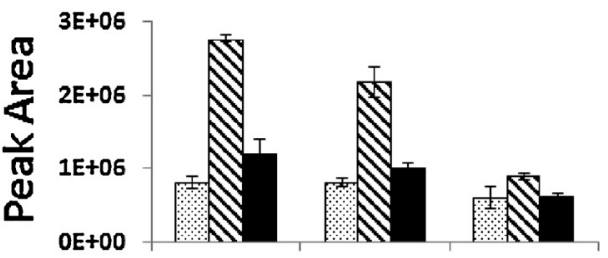

E1

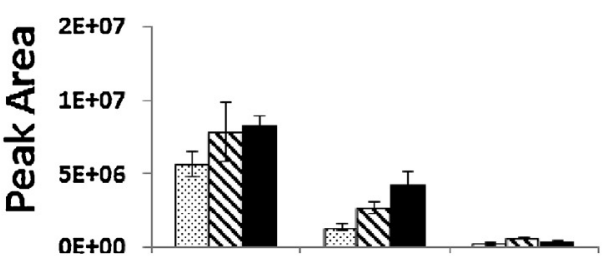

Endrin

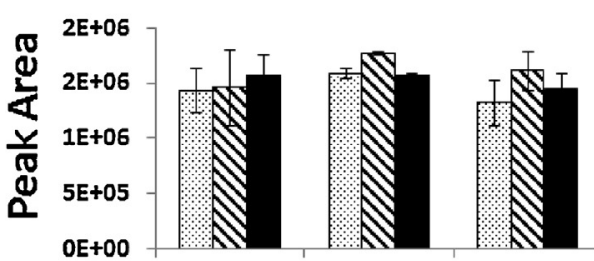

Acenaphthene

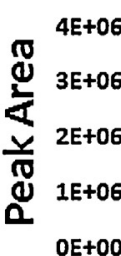

TCS

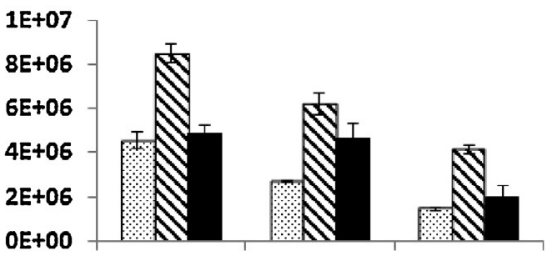

2-OHBP

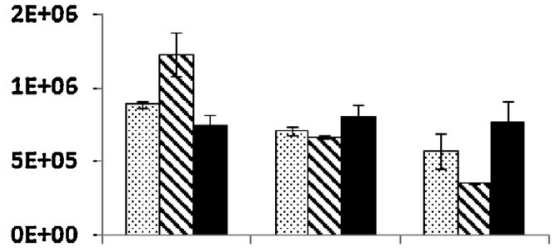

Cyfluthrins

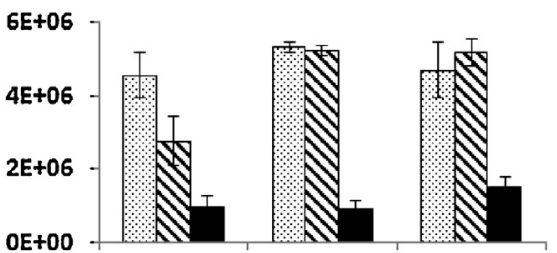

Ethion

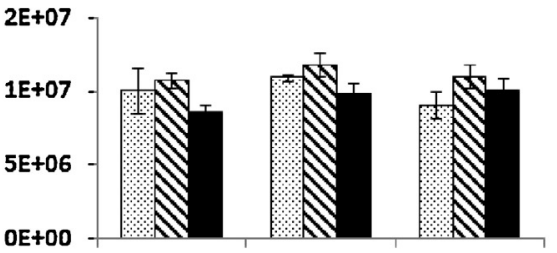

Pyrene

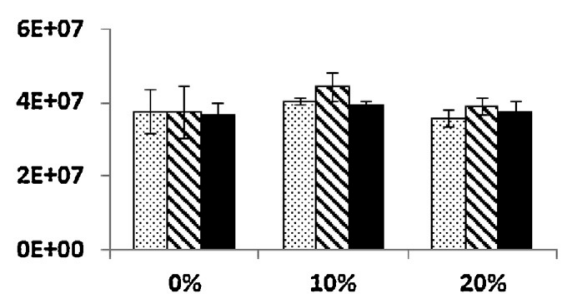

4-MBC

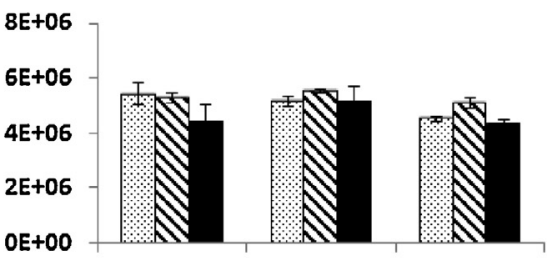

Nonylphenol

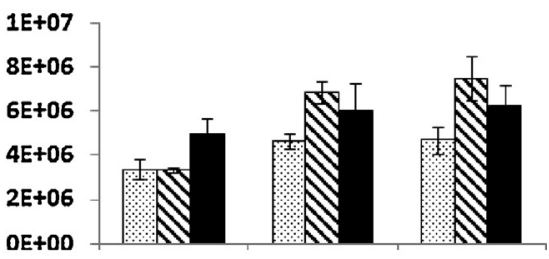

PCB 138

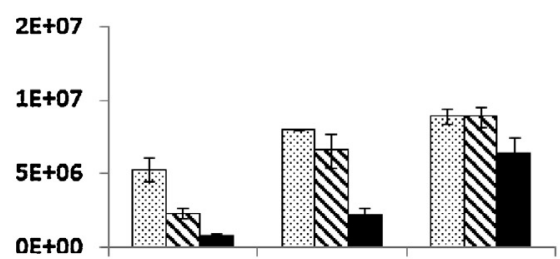

Terbuthylazine

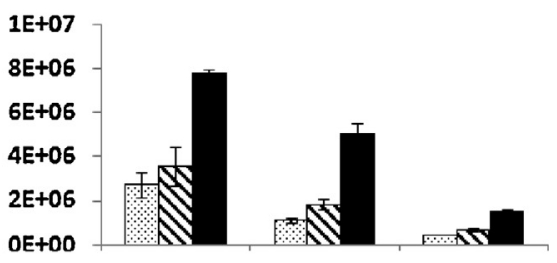

Dibenzo(a,h)anthracene

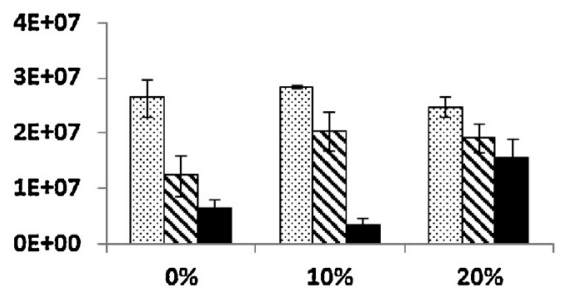

\section{$0 \mathrm{~g} / \mathrm{l} \mathbb{\mathbb { 1 }} 100 \mathrm{~g} / \mathrm{l} \square 200 \mathrm{~g} / \mathrm{l}$}

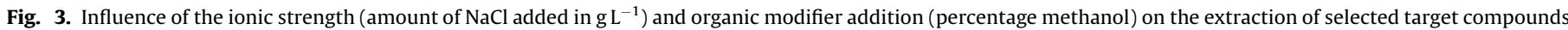
by SBSE.

optimized conditions (agitation time $=5 \mathrm{~h}, \mathrm{NaCl}=100 \mathrm{~g} \mathrm{~L}^{-1}$, no methanol addition, sample volume $=100 \mathrm{~mL}, \mathrm{Na}_{2} \mathrm{CO}_{3}=10 \mathrm{~g} \mathrm{~L}^{-1}$, acetic anhydride $=500 \mu \mathrm{L}, 10 \mathrm{~mm}$ length bars) recoveries were: up to $98 \%$ for PAHs (the lowest recoveries values, $<30 \%$, for the heaviest compounds such as dibenzo[ $a, h]$ anthracene or benzo[g, $h$, i]perylene); up to $93 \%$ for pesticides (e.g., $83 \%$ for triazines, $93 \%$ for organochlorines, $80 \%$ for organophosphates, and $75 \%$ for triazines); and up to $95 \%$ for emerging contaminants (e.g., $35-85 \%$ for endocrine disruptor compounds and 52-95\% for fragrances).

\subsection{GC-APGC-ToF-MS analysis}

Table 1 shows target compounds, retention times and accurate mass of ions $\left([\mathrm{M}+\mathrm{H}]^{+}\right.$in most cases) obtained using GC-APGC-ToF- 


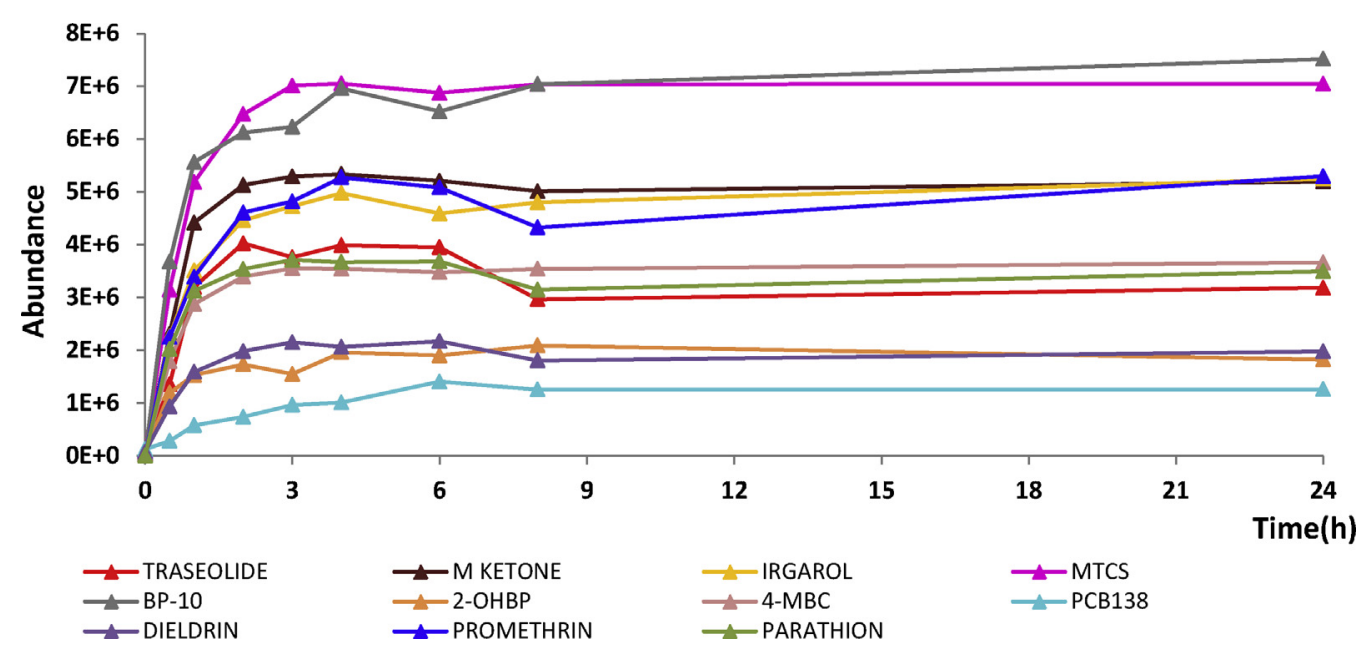

Fig. 4. Evolution in the signal intensities of selected target compounds after performing SBSE at different time periods (from 1 to $24 \mathrm{~h}$ ).

MS. Analyses were carried out in full-scan mode. First, we compared differences in the ionization process between APGC and most widely used EI. As an example, the macrocyclic fragrance Musk R1 was fragmented into many different ions using EI (Fig. 5A). The molecular ion $(\mathrm{m} / \mathrm{z}=257)$ could not be detected and some of the $m / z$ ratios in the mass spectrum of these chemical (e.g., $m / z=98,169$ and 241) were also common for other target (e.g., $\mathrm{BP} 10, \beta$ endosulfan, prometryn) and non-target compounds, which could lead to the occurrence false positives in environmental samples. We decide to use $m / z=169$ as the main fragment for the identification and quantification of Musk R1, corresponding to the fragmentation at the ester bond and loss of 87 mass units, $\mathrm{CH}_{3}$ $\left(\mathrm{CH}_{2}\right)_{4} \mathrm{O}^{*}$. When APGC is used instead, ionization is heavily influenced by the presence of water traces in the source that enhance protonation processes. This results in a "softer" ionization that promotes the formation of $[\mathrm{M}+\mathrm{H}]^{+}$ions for most compounds $[19,36]$, including Musk R1, where $m / z=257$ shows now the highest intensity (Fig. 5B). Some exceptions were helvetolide,
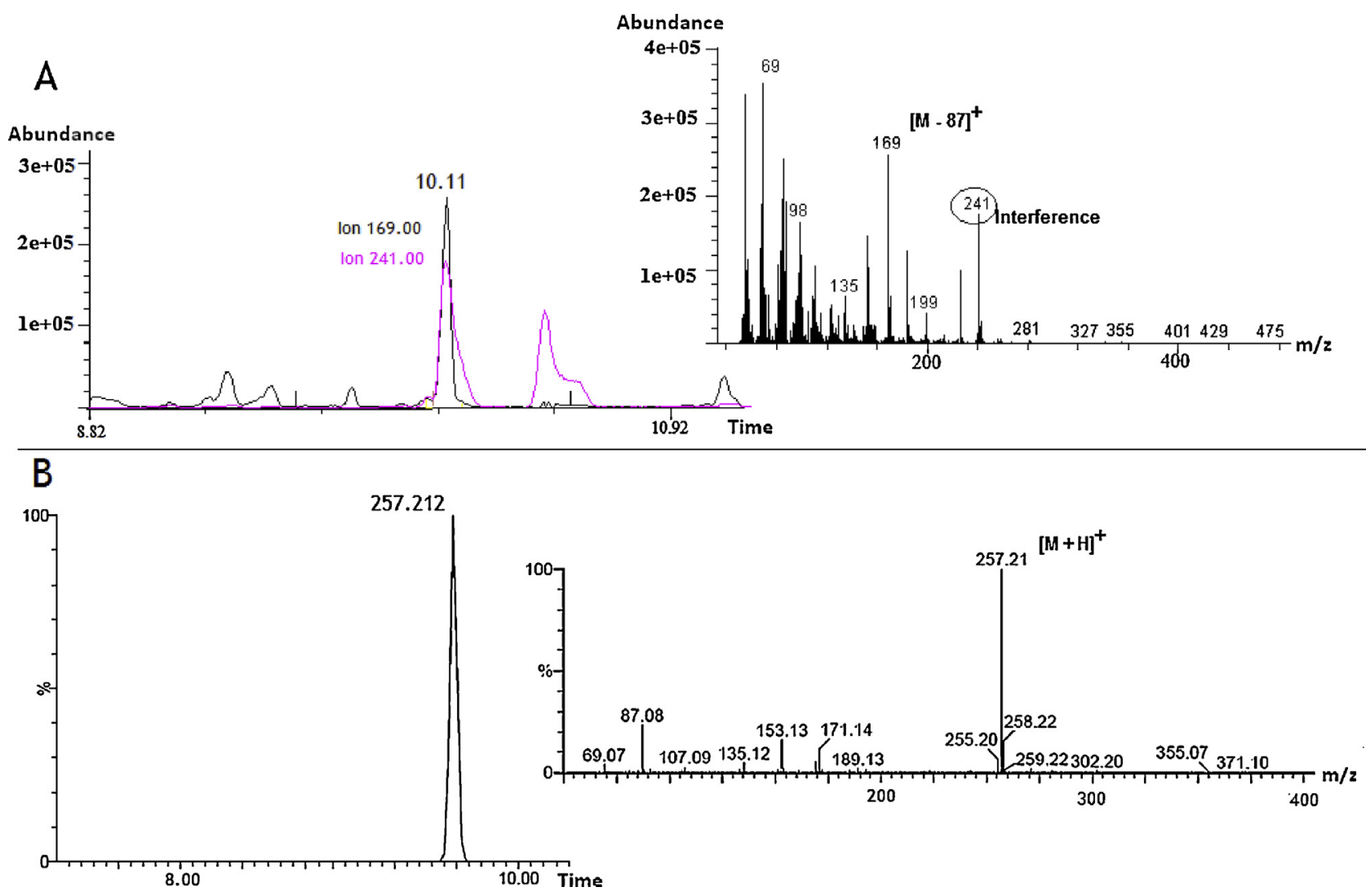

Fig. 5. Extracted ion chromatograms (left) and mass spectra (right) for Musk R1 using GC-EI-Q-MS (A) and GC-APGC-ToF-MS (B). 

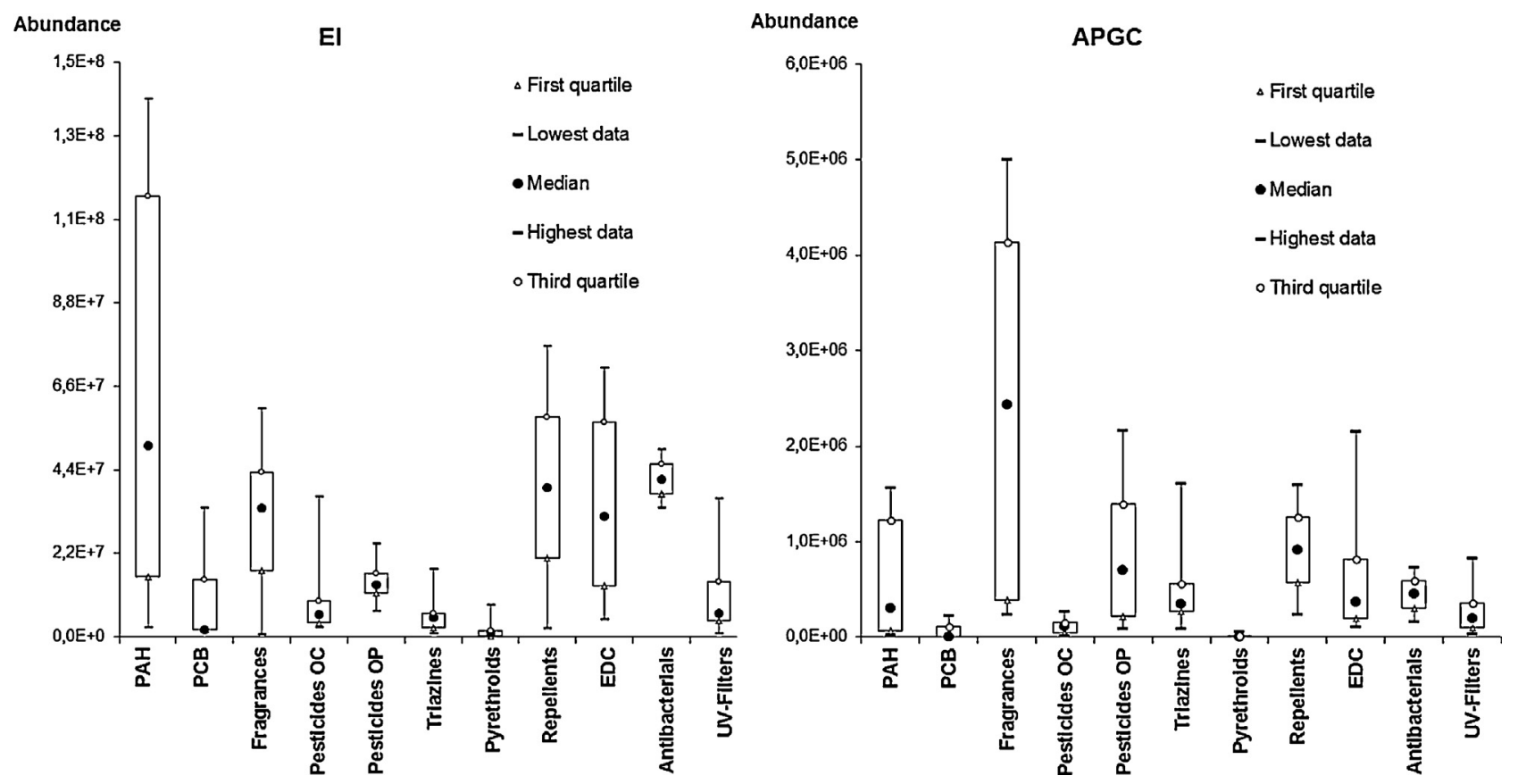

Fig. 6. MS signal intensities for different groups of target compounds after ionization by EI and APGC.

galaxolide and BS, where the most abundant specific fragment ions were selected instead (Table 1 ).

Fig. 6 provides an illustrative example of the efficiency of both EI and APGC sources for the ionization of contaminants. The first source, EI, is a good option when working with very stable compounds such as PAHs, which require higher ionization energies for ionization and fragmentation. On the other hand, better results were obtained using APGC for more labile compounds (e.g., fragrances). Even if signal intensities were comparable between the two sources, APGC is still a better option for some specific compounds such as pyrethroids, which are broken into a myriad of non-specific fragments by EI, therefore making their identification in complex environmental matrices more challenging [37]. Additionally, APGC allows progressive fragmentation of analytes by applying different cone voltages. Two different functions were selected ( 10 and $40 \mathrm{eV}$ ) in our standard MS method to guarantee accurate identification and quantification of target compounds. This is especially critical for some macrocyclic fragrances which could not be properly determined by EI due to the co-elution of impurities showing the same common fragment ions. As an example, Fig. 7 shows two mass spectra at different cone voltages for muscone. The lower energy spectrum $(10 \mathrm{eV})$ was employed for quantification of this target compound, as the main ion 239, corresponding to $[\mathrm{M}+\mathrm{H}]^{+}$, showed the highest intensity (Fig. 7A). The higher energy spectrum $(40 \mathrm{eV})$ allowed further fragmentation of the molecule (Fig. 7B). Then, a specific software (Mass Fragment) was used to study different fragmentation patterns and to identify possible specific fragment ions for muscone.

EI mass spectra of regulated and some emerging compounds can be found at commercially available libraries, such as NIST. This allows identification of target (and non-target) chemicals by comparison of spectra in full-scan mode. However, this task becomes more challenging or impossible when trace analysis is performed, as many other co-eluting compounds at higher concentrations than the analytes can interfere. APGC, in combination with time-of-flight (ToF) mass spectrometry can be used as an alternative for the identification of compounds. This is based on the accurate mass measurements of the protonated or deprotonated molecules and/or specific fragments ions. Fig. 8 shows some illustrative examples of the applicability of this technique for target (and non-target) analysis of hydrophobic microcontaminants in water samples. Triclosan (TCS) is an antimicrobial found in surface water samples. We could confirm its presence by comparing its retention time $(13.5 \mathrm{~min})$ and accurate mass measurement (error $<0.3 \mathrm{~m} \mathrm{Da}$ ) of its molecular ion with those for a standard. Accurate mass measurement and the presence of chlorine atoms in the TCS molecule allow using an additional confirmation criterion, which consists of comparing the original acquired mass spectrum with the theoretical isotope model based on the chemical formula of the compound. Elemental composition of non-target compounds can be also obtained by accurate mass measurement in full-mode scan. Thus, we were able to identify the presence of organophosphorus flame retardants such as tributhyl phosphate (TBP) (Fig. 8) in the same sample in which TCS was detected, and later confirm its identity by matching its retention time with that for a commercial standard. More examples on the identification of non-target compounds are shown in Supplementary Information.

\subsection{Analytical performance of the method and analysis of real samples}

The MS response of all compounds was linear between 0.001 to $1 \mu \mathrm{g} \mathrm{L}^{-1}$ and coefficients of determination $\left(R^{2}\right)$ (Table 1 ) for calibration curves were always above 0.9 for target compounds, and over 0.96 for $75 \%$ of them. Reproducibility and repeatability of the method showed resulting SDs (standard deviations) below $20 \%$ for most of the target analytes. The method detection limits (MDL) ranged from 0.02 to $18 \mathrm{ng} \mathrm{L}^{-1}$ and were below $1 \mathrm{ng} \mathrm{L}^{-1}$ for more than $70 \%$ of the chemicals tested (Table 1 ). Most analytes were not detected in blanks performed with HPLC water, except for some compounds such as galaxolide, EHMC and OC, which presented occasional contamination problems $[28,38]$. SBSE bars were cleaned repeatedly in order to minimize this issue. Matrix effect was also studied by spiking real samples and HPLC water with a mix of deuterated compounds at $0.1 \mu \mathrm{gL}^{-1}$. The MS signal intensities of these surrogates were compared with those obtained 


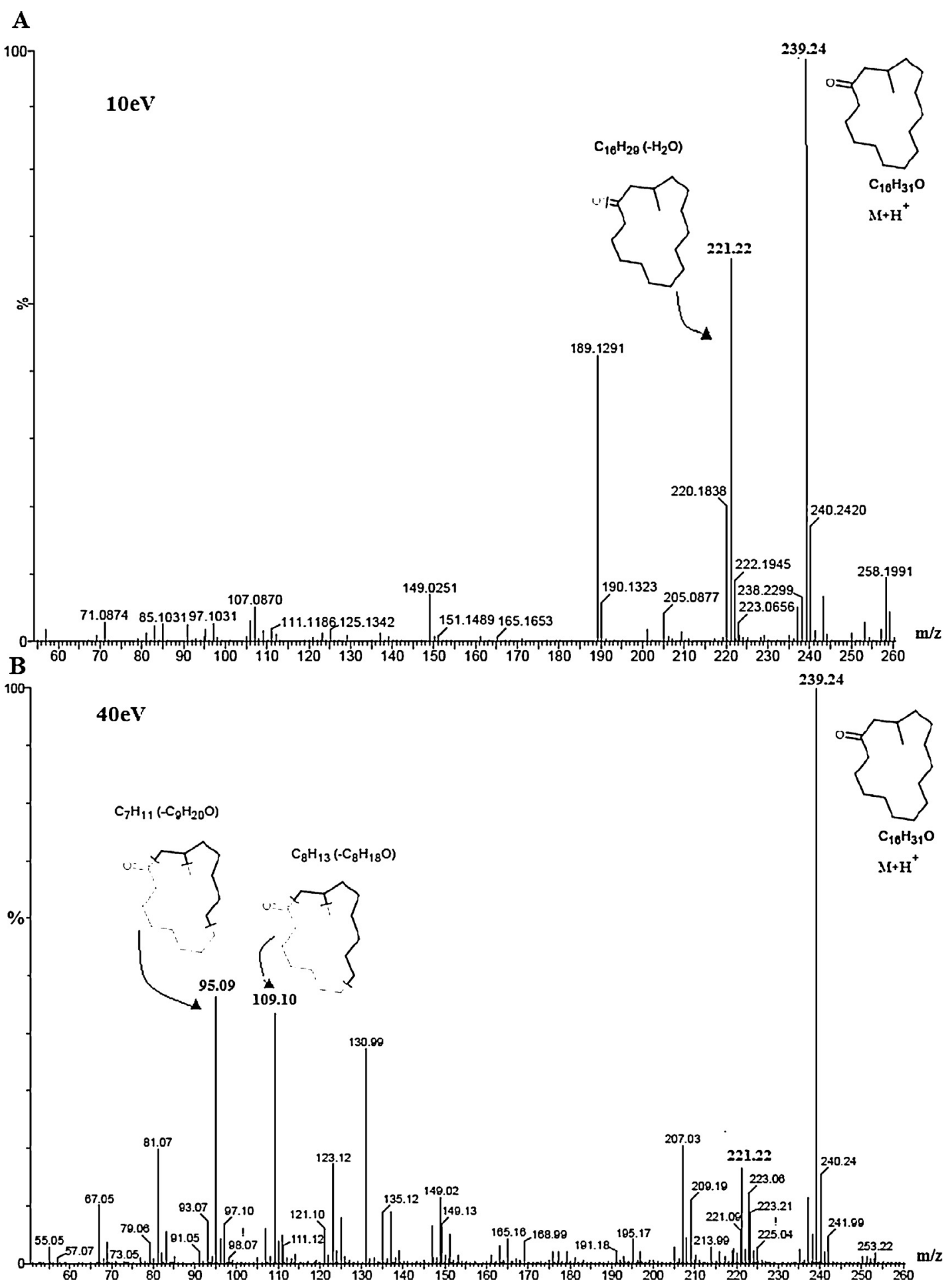

Fig. 7. Low ( $10 \mathrm{eV})(\mathrm{A})$ and high $(40 \mathrm{eV})$ (B) energy APGC-ToF-MS spectra for muscone. Tentative structures for different ion fragments are also shown.

from real sample extracts. Ionization efficiency did not decrease dramatically in any environmental sample, only up to $30 \%$ in the dirtiest extracts corresponding to effluent water samples. According to De Sousa and co-workers [39], presence of other co-eluting compounds could also affect the retention time of the analytes, although this was not observed in our case.

Once the method was developed, it was applied to the analysis of target compounds in several matrices sampled within the province of Cadiz (SW of Spain): effluent, river water, seawater and groundwater. These samples were extracted and analyzed in triplicate to determine the reproducibility of the method. Results are shown in Table 2. Twenty one out of 102 target compounds were detected and quantified. A few of them, mostly personal care products (OTNE, celestolide, HHCB, MK, BP-3, TCS, HMS, 4-MBC, OC and $\mathrm{EHMC}$ ) were quantified in all samples. As expected from sewage-derived contaminants, the highest concentrations were measured in the effluent of the WWTP, whereas the lowest values were observed in groundwater and seawater. Concentrations in 


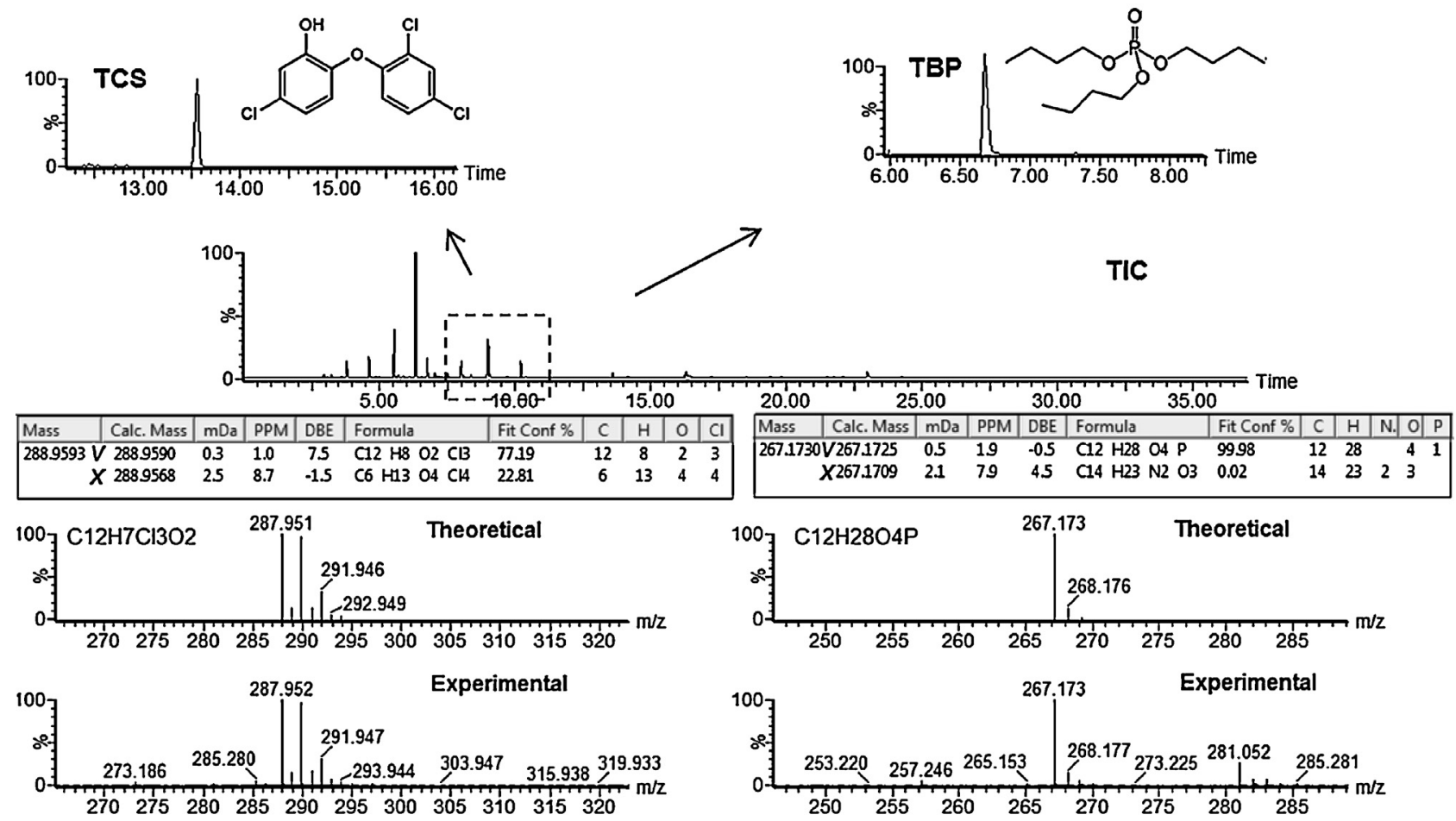

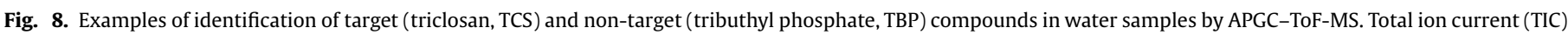
chromatogram, mass spectra, elemental composition and isotopic distributions are shown.

effluent samples for some UV-filters such as BP-3 or EHMC were lower than previously reported by other authors [22], probably due to the sampling season being fall instead of summer, when these compounds are more intensively used. Most of the regulated compounds (PCBs, organochlorine pesticides, etc.) could not be detected in any sample, with the exception of some pesticides such as chlorpyrifos or terbuthylazine, which were detected in groundwater from an agricultural area where these chemicals are often applied.

Table 2

Concentrations of target compounds (ng L $\mathrm{L}^{-1}$ ) and standard deviations (SDs) in different environmental matrices $(n=3)$ determined by GC-APGC-ToF-MS analysis $(<\mathrm{MDL}=$ below method detection limits)

\begin{tabular}{lllll}
\hline Target compounds & Effluent & River water & Sea water & Ground water \\
\hline DEET & $560 \pm 160$ & $25 \pm 10$ & $<\mathrm{MDL}$ & $<\mathrm{MDL}$ \\
Irgarol & $4 \pm 1$ & $<\mathrm{MDL}$ & $<\mathrm{MDL}$ & $<\mathrm{MDL}$ \\
Cashmeran & $230 \pm 20$ & $36 \pm 3$ & $<\mathrm{MDL}$ & $3 \pm 0$ \\
OTNE & $17,800 \pm 2240$ & $5460 \pm 500$ & $400 \pm 10$ & $520 \pm 30$ \\
Celestolide & $17 \pm 1$ & $5 \pm 0$ & $2 \pm 0$ & $2 \pm 0.5$ \\
Galaxolide & $4750 \pm 800$ & $1890 \pm 100$ & $<\mathrm{MDL}$ & $240 \pm 20$ \\
Tonalide & $780 \pm 70$ & $190 \pm 20$ & $<\mathrm{MDL}$ & $22 \pm 4$ \\
Musk ketone & $210 \pm 40$ & $160 \pm 20$ & $<\mathrm{MDL}$ & $<\mathrm{MDL}$ \\
BP-3 & $82 \pm 7$ & $66 \pm 1$ & $60 \pm 8$ & $63 \pm 5$ \\
BpA & $65 \pm 3$ & $65 \pm 2$ & $<\mathrm{MDL}$ & $<\mathrm{MDL}$ \\
TCS & $95 \pm 8$ & $72 \pm 0.5$ & $66 \pm 1$ & $68 \pm 1$ \\
MTCS & $52 \pm 1$ & $<\mathrm{MDL}$ & $<\mathrm{MDL}$ & $<\mathrm{MDL}$ \\
EHS & $3 \pm 1$ & $<\mathrm{MDL}$ & $<\mathrm{MDL}$ & $<\mathrm{MDL}$ \\
HMS & $22 \pm 5$ & $8 \pm 0.5$ & $9 \pm 2$ & $11 \pm 0.7$ \\
$4-$ MBC & $49 \pm 1$ & $46 \pm 2$ & $46 \pm 4$ & $46 \pm 6$ \\
E1 & $61 \pm 0.5$ & $62 \pm 0$ & $<\mathrm{MDL}$ & $<\mathrm{MDL}$ \\
EHMC & $31 \pm 6$ & $30 \pm 1$ & $36 \pm 1.5$ & $43 \pm 10$ \\
OC & $56 \pm 4$ & $32 \pm 1$ & $49 \pm 3$ & $59 \pm 8$ \\
Pyrene & $3 \pm 1$ & $<\mathrm{MDL}$ & $<\mathrm{MDL}$ & $<\mathrm{MDL}$ \\
Terbuthylazine & $<\mathrm{MDL}$ & $<\mathrm{MDL}$ & $<\mathrm{MDL}$ & $300 \pm 9$ \\
Chlorpyrifos & $200 \pm 8$ & $20 \pm 4$ & $<\mathrm{MDL}$ & $10 \pm 2$ \\
\hline & & & & \\
\hline & & & & \\
\hline
\end{tabular}

\section{Conclusions}

This work presents SBSE-LD combined with APGC-ToF-MS as a new powerful tool for the analysis of target (and non-target) regulated and emerging microcontaminants in aqueous samples. This methodology allows the simultaneous extraction and determination of a wide number of analytes in environmental water samples in one single run. It requires relatively little sample manipulation, reduced extraction times compared to other protocols that often rely on subsequent solvent evaporation steps (e.g., liquid-liquid or solid phase extractions), and also allows reutilization of PDMS bars. APGC-ToF-MS can be used as an alternative analytical tool over more conventional GC-EI-MS, especially for more labile compounds (e.g., fragrances or pyrethroids) that are better detected and identified when lower ionization energies are used. Although MS spectrum libraries are not available for APGC, identification of target and non-target compounds at trace levels is feasible by accurate measurement of molecular ions using ToF detectors. As an example, new organophosphorus flame retardants were detected for the first time in water samples from the province of Cadiz. Regarding the occurrence of target compounds in this area, 21 out of 102 chemicals were measured at concentrations up to $17.8 \mu \mathrm{g} \mathrm{L}^{-1}$, 18 of them belonging to the group of personal care products.

\section{Acknowledgements}

This study has been carried out by the support of two regional research projects (RNM 5417 and RNM 6613), and with the help of a Spanish Ministry of Education, Culture and Sport grant. We express our gratitude to E. de Miguel (SCCYT, University of Cádiz) for his technical support with the APGCMS/Q-TOF system. 


\section{Appendix A. Supplementary data}

Supplementary data associated with this article can be found, in the online version, at http://dx.doi.org/10.1016/j.aca.2014.05.030.

\section{References}

[1] M.J. Gomez, S. Herrera, D. Sole, E. Garcia-Calvo, A.R. Fernandez-Alba, Automatic searching and evaluation of priority and emerging contaminants in wastewater and river water by stir bar sorptive extraction followed by comprehensive two-dimensional gas chromatography-time-of-flight mass spectrometry, Anal. Chem. 83 (2011) 2638-2647.

[2] A. Johnsen, U. Karlson, Diffuse PAH contamination of surface soils: environmental occurrence, bioavailability, and microbial degradation, Appl. Microbiol. Biotechnol. 76 (2007) 533-543.

[3] Stockholm convention on persistent organic pollutants. http://www.wipo.int wipolex/en/other_treaties/text.jsp?file_id=194841, 2001.

[4] M.G. Pintado-Herrera, E. Gonzalez-Mazo, P.A. Lara-Martin, Determining the distribution of triclosan and methyl triclosan in estuarine settings, Chemosphere 95 (2013) 478-485.

[5] V.L.B. Jaspers, C. Sonne, F. Soler-Rodriguez, D. Boertmann, R. Dietz, M. Eens, L. M. Rasmussen, A. Covaci, Persistent organic pollutants and methoxylated polybrominated diphenyl ethers in different tissues of white-tailed eagles (Haliaeetus albicilla) from West Greenland, Environ. Pollut. 175 (2013) 137-146.

[6] S. O'Toole, C. Metcalfe, Synthetic musks in fish from urbanized areas of the lower Great Lakes, Canada, J. Great Lakes Res. 32 (2006) 361-369.

[7] P. Gago-Ferrero, M. Diaz-Cruz, D. Barcelo, An overview of UV-absorbing compounds (organic UV filters) in aquatic biota, Anal. Bioanal. Chem. 404 (2012) 2597-2610.

[8] European water policy directive 2008/105/EC. http://eur-lex.europa.eu/ LexUriServ/LexUriServ.do?uri=CELEX:32008L0105:EN:NOT, 2008.

[9] A. Azzouz, B. Souhail, E. Ballesteros, Continuous solid-phase extraction and gas chromatography-mass spectrometry determination of pharmaceuticals and hormones in water samples, J. Chromatogr. A 1217 (2010) 2956-2963.

[10] O. Krüger, G. Christoph, U. Kalbe, W. Berger, Comparison of stir bar sorptive extraction (SBSE) and liquid-liquid extraction (LLE) for the analysis of polycyclic aromatic hydrocarbons (PAH) in complex aqueous matrices, Talanta 85 (2011) 1428-1434.

[11] H. Liu, L. Liu, Y. Xiong, X. Yang, T. Luan, Simultaneous determination of UV filters and polycyclic musks in aqueous samples by solid-phase microextraction and gas chromatography-mass spectrometry, J. Chromatogr. A 1217 (2010) 6747-6753.

[12] N. Barco-Bonilla, R. Romero-González, P. Plaza-Bolaños, J.L. FernándezMoreno, A. Garrido Frenich, J.L. Martínez Vidal, Comprehensive analysis of polycyclic aromatic hydrocarbons in wastewater using stir bar sorptive extraction and gas chromatography coupled to tandem mass spectrometry, Anal. Chim. Acta 693 (2011) 62-71.

[13] M. Kawaguchi, R. Ito, H. Honda, N. Endo, N. Okanouchi, K. Saito, Y. Seto, H. Nakazawa, Simultaneous analysis of benzophenone sunscreen compounds in water sample by stir bar sorptive extraction with in situ derivatization and thermal desorption-gas chromatography-mass spectrometry, J. Chromatogr. A 1200 (2008) 260-263.

[14] A. Prieto, O. Basauri, R. Rodil, A. Usobiaga, L.A. Fernández, N. Etxebarria, O. Zuloaga, Stir-bar sorptive extraction: a view on method optimisation, nove applications, limitations and potential solutions, J. Chromatogr. A 1217 (2010) 2642-2666.

[15] E. Pérez-Carrera, V.M. León, A. Parra Gómez, E. González-Mazo, Simultaneous determination of pesticides, polycyclic aromatic hydrocarbons and polychlorinated biphenyls in seawater and interstitial marine water samples, using stir bar sorptive extraction-thermal desorption-gas chromatography-mass spectrometry, J. Chromatogr. A 1170 (2007) 82-90.

[16] M.G. Pintado-Herrera, E. Gonzalez-Mazo, P.A. Lara-Martin, Environmentally friendly analysis of emerging contaminants by pressurized hot water extraction-stir bar sorptive extraction-derivatization and gas chromatography-mass spectrometry, Analytical and Bioanalytical Chemistry 405 (2013) 401-411.

[17] J.B. Quintana, R. Rodil, S. Muniategui-Lorenzo, P. López-Mahía, D. PradaRodríguez, Multiresidue analysis of acidic and polar organic contaminants in water samples by stir-bar sorptive extraction-liquid desorption-gas chromatography-mass spectrometry, J. Chromatogr. A 1174 (2007) 27-39.

[18] S. Machado, C. GonÇalves, E. Cunha, A. Guimaraes, M.F. Alpendurada, New developments in the analysis of fragrances and earthy-musty compounds in water by solid-phase microextraction (metal alloy fibre) coupled with gas chromatography (tandem) mass spectrometry, Talanta 84 (2011) 1133-1140.

[19] E. Canellas, P. Vera, C. Domeño, P. Alfaro, C. Nerín, Atmospheric pressure gas chromatography coupled to quadrupole-time of flight mass spectrometry as a powerful tool for identification of non intentionally added substances in acrylic adhesives used in food packaging materials, J. Chromatogr. A 1235 (2012) 141-148.

[20] T. Portolés, L. Cherta, J. Beltran, F. Hernández, Improved gas chromatographytandem mass spectrometry determination of pesticide residues making use of atmospheric pressure chemical ionization, J. Chromatogr. A 1260 (2012) 183-192.

[21] D. Stevens, Q. Shi, C.S. Hsu, Novel analytical technique for petroleum biomarker analysis, Energ. Fuel. 27 (2013) 167-171.

[22] E. Magi, M. Di Carro, C. Scapolla, K.T.N. Nguyen, Stir Bar Sorptive Extraction and LC-MS/MS for trace analysis of UV filters in different water matrices, Chromatographia (2012) 1-10.

[23] H. Kataoka, Derivatization reactions for the determination of amines by gas chromatography and their applications in environmental analysis, J. Chromatogr. A 733 (1996) 19-34.

[24] K.D. Buchholz, J. Pawllszyn, Optimization of solid -phase microextraction conditions for determination of phenols, Anal. Chem. 66 (1994) 160-167.

[25] K.K. Stenerson, The derivatization and analysis of amino acids by GC-MS. Reporter US. 25.3. http://www.sigmaaldrich.com/content/dam/sigma-aldrich/ articles/reporter-us/pdf/the-derivatization.pdf, 2011.

[26] M. Kawaguchi, Y. Ishii, N. Sakui, N. Okanouchi, R. Ito, K. Inoue, K. Saito, H. Nakazawa, Stir bar sorptive extraction with in situ derivatization and thermal desorption-gas chromatography-mass spectrometry in the multi-shot mode for determination of estrogens in river water samples, J. Chromatogr. A 1049 (2004) 1-8.

[27] J. Kumirska, A. Plenis, P. Lukaszewicz, M. Caban, N. Migowska, A. BialkBielinska, M. Czerwicka, P. Stepnowski, Chemometric optimization of derivatization reactions prior to gas chromatography-mass spectrometry analysis, J. Chromatogr. A 1296 (2013) 164-178.

[28] N. Negreira, I. Rodriguez, E. Rubi, R. Cela, Dispersive liquid-liquid microextraction followed by gas chromatography-mass spectrometry for the rapid and sensitive determination of UV filters in environmental water samples, Anal. Bioanal. Chem. 398 (2010) 995-1004.

[29] N. Ramírez, R.M. Marcé, F. Borrull, Development of a stir bar sorptive extraction and thermal desorption-gas chromatography-mass spectrometry method for determining synthetic musks in water samples, J. Chromatogr. A 1218 (2011) $156-161$.

[30] A. Sanchez-Ortega, N. Unceta, A. Gómez-Caballero, M.C. Sampedro, U. Akesolo, M.A. Goicolea, R.J. Barrio, Sensitive determination of triazines in underground waters using stir bar sorptive extraction directly coupled to automated thermal desorption and gas chromatography-mass spectrometry, Anal. Chim. Acta 641 (2009) 110-116.

[31] M. Arbulu, M.C. Sampedro, N. Unceta, A. Gómez-Caballero, M.A. Goicolea, R.J. Barrio, A retention time locked gas chromatography-mass spectrometry method based on stir-bar sorptive extraction and thermal desorption for automated determination of synthetic musk fragrances in natural and wastewaters, J. Chromatogr. A 1218 (2011) 3048-3055.

[32] F.J. Camino-Sánchez, A. Zafra-Gómez, J.P. Pérez-Trujillo, J.E. Conde-González, J. C. Marques, J.L. Vílchez, Validation of a GC-MS/MS method for simultaneous determination of 86 persistent organic pollutants in marine sediments by pressurized liquid extraction followed by stir bar sorptive extraction, Chemosphere 84 (2011) 869-881.

[33] N. Ochiai, K. Sasamoto, H. Kanda, E. Pfannkoch, Sequential stir bar sorptive extraction for uniform enrichment of trace amounts of organic pollutants in water samples, J. Chromatogr. A 1200 (2008) 72-79.

[34] M.C. Sampedro, M.A. Goicolea, N. Unceta, A. Sanchez-Ortega, R.J. Barrio, Sequential stir bar extraction, thermal desorption and retention time locked GC-MS for determination of pesticides in water, J. Sep. Sci. 32 (2009) 3449-3456.

[35] J.I. Cacho, N. Campillo, P. Viñas, M. Hernández-Córdoba, Determination of alkylphenols and phthalate esters in vegetables and migration studies from their packages by means of stir bar sorptive extraction coupled to gas chromatography-mass spectrometry, J. Chromatogr. A 1241 (2012) 21-27.

[36] C. Domeño, E. Canellas, P. Alfaro, A. Rodriguez-Lafuente, C. Nerin, Atmospheric pressure gas chromatography with quadrupole time of flight mass spectrometry for simultaneous detection and quantification of polycyclic aromatic hydrocarbons and nitro-polycyclic aromatic hydrocarbons in mosses, J. Chromatogr. A 1252 (2012) 146-154.

[37] F.A. Esteve-Turrillas, A. Pastor, M. de la Guardia, Comparison of different mass spectrometric detection techniques in the gas chromatographic analysis of pyrethroid insecticide residues in soil after microwave-assisted extraction, Anal. Bioanal. Chem. 384 (2006) 801-809.

[38] R. Rodil, M. Moeder, Development of a method for the determination of UV filters in water samples using stir bar sorptive extraction and thermal desorption-gas chromatography-mass spectrometry, J. Chromatogr. A 1179 (2008) 81-88.

[39] F.A. de Sousa, A.I.G. Costa, M. de Queiroz, R.F. Teofilo, A.A. Neves, G.P. de Pinho, Evaluation of matrix effect on the GC response of eleven pesticides by PCA, Food Chem. 135 (2012) 179-185. 